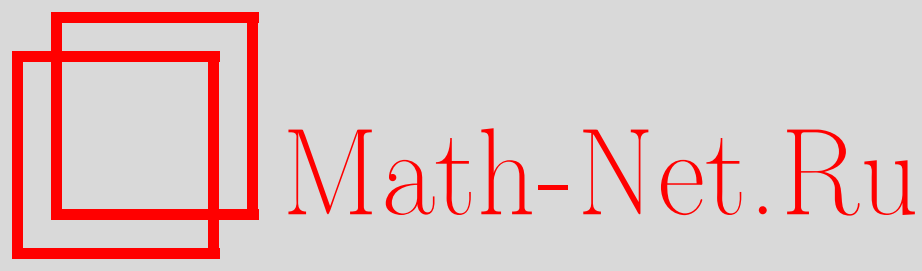

А. Ю. Морозов, А. А. Морозов, А. В. Пополитов, Матричные модели и размерности в вершинах гиперкубов, TMФ, 2017, том 192, номер 1, 115-163

DOI: https://doi.org/10.4213/tmf9214

Использование Общероссийского математического портала Math-Net.Ru подразумевает, что вы прочитали и согласны с пользовательским соглашением http://www . mathnet.ru/rus/agreement

Параметры загрузки:

IP : 54.81 .137 .203

26 апреля 2023 г., 11:40:20

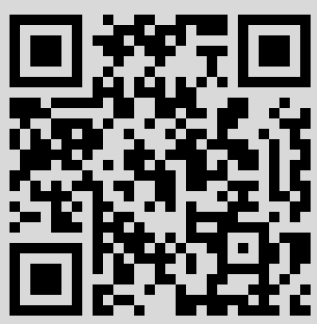




\title{
ФИЗИКА
}

Том 192, № 1

июль, 2017

(C) 2017 г.
А. Ю. Морозов ${ }^{* \dagger}$, А. А. Морозов ${ }^{* \dagger \ddagger \S, ~}$ А. В. Пополитов ${ }^{* \dagger}$

\section{МАТРИЧНЫЕ МОДЕЛИ И РАЗМЕРНОСТИ В ВЕРШИНАХ ГИПЕРКУБОВ}

\begin{abstract}
Рассматриваются корреляционные функции в теории Черна-Саймонса (полиномы узлов). Используется подход, в котором каждая диаграмма узла ассоциируется с гиперкубом. При этом основную роль играет число циклов, на которые диаграмма разбивается при различных разрешениях. Некоторые функции от этих чисел далее можно интерпретировать как размерности градуированных пространств, связанных с вершинами гиперкубов, но поиск этих функций является весьма нетривиальной задачей. Ранее было высказано предложение решать эту проблему с помощью методов теории матричных моделей по аналогии с топологической рекурсией. Предлагается развитие этой идеи и приводится широкий набор нетривиальных примеров, связанных как с обычными, так и с виртуальными узлами и зацеплениями. Наиболее мощная версия формализма свободно связывает обычные узлы/зацепления с виртуальными; более того, она позволяет продвинуться за пределы связанного с узлами множества $(2,2)$-валентных графов.
\end{abstract}

Ключевые слова: теория Черна-Саймонса, теория узлов, виртуальные узлы, матричные модели.

DOI: https://doi.org/10.4213/tmf9214

\section{1. ВВЕДЕНИЕ}

Полиномы узлов [1] (вильсоновские средние в теории Черна-Саймонса [2] и их деформации [3]-[10]) в данный момент являются одной из активно развивающихся областей теоретической физики. Они принадлежат к важному классу $\tau$-функций

Работа выполнена в Институте проблем передачи информации им. А. А. Харкевича РАН при поддержке Российского научного фонда (грант 14-50-00150).

${ }^{*}$ Институт теоретической и экспериментальной физики, Москва, Россия.

E-mail: morozov@itep.ru, Andrey.Morozov@itep.ru

${ }^{\dagger}$ Институт проблем передачи информации им. А. А. Харкевича РАН, Москва, Россия

‡Национальный исследовательский ядерный университет "МИФИ", Москва, Россия

$\S$ Лаборатория квантовой топологии, Челябинский государственный университет, Челябинск, Россия

『Korteweg-de Vries Institute for Mathematics, University of Amsterdam, Amsterdam, The Netherlands 
Гурвица [11] и предположительно обладают основными свойствами матрично-модельных $\tau$-функций [12], [13], которые сейчас объединяют все известные специальные функции и дают основу для аналитического описания природы.

Существует несколько способов изучения и даже определения полиномов узлов, связанных с разными пересекающимися областями физики и математики, которые должны быть соотнесены друг с другом: теория представлений, топология, модулярные преобразования конформных блоков, интегрируемые системы, теория Морзе и т. д. Самая интригующая из этих областей - это программа категорификации [14], в этом контексте впервые предложенная Ховановым [4]. Физическая интерпретация этой программы состоит в переходе к пространствам более высоких размерностей (где интересующие нас величины связаны с устойчивыми точками некоторой новой эволюции [15]). Детальное описание данных идей находится за пределами рассмотрения настоящей статьи. Здесь мы сконцентрируемся на технических подробностях, необходимых для построения такого формализма и проведения эффективных расчетов в его рамках. Для этих целей мы опираемся на пару относительно старых достижений - подходе гиперкуба [16]-[19] и его значимого упрощения из работы [20] вкупе с недавно предложенной матрично-модельной интерпретацией [21]. Это практически позволило нам решить задачу вычисления квантовых размерностей "пространств" в вершинах гиперкубов, которые являются сложными составляющими полиномов ХОМФЛИ. Данный результат открывает нам путь к простой интерпретации морфизмов разрезания и склейки и далее к простому вычислению гомологий, позволяя получить суперполиномы Хованова-Рожанского, хотя эту часть истории мы оставим для будущих рассмотрений.

Тем не менее уже в данном тексте мы описываем новые многообещающие соотношения между различными ветвями теории узлов и устанавливаем новые связи с другими областями математической физики. Наиболее примечательно то, что похожая на матрично-модельную рекурсию процедура вначале оказывается напрямую связанной с инвариантностью относительно движений Рейдемейстера, если ограничить рассмотрение обычными узлами, а в общем случае не только смешивает обычные узлы с виртуальными, но и обобщает рассмотрение на теорию графов с валентностью много больше четырех. Замечательно, что это не только привлекательный плод фантазии или взгляд на теорию (каковым он был в оригинальных работах [22] и [20]), но и необходимый для вычисления метод, использующийся в рабочей компьютерной программе, цель которой - вычисление полиномов узлов с помощью подхода гиперкуба. Будущие работы в этом направлении несомненно приведут к новым достижениям, как концептуальным, так и практическим.

\section{2. ПОДХОД ДОЛОТИНА-МОРОЗОВА}

В данном разделе мы напомним основные компоненты новой версии [20] подхода гиперкуба [16]-[19], изучение которого было продолжено в [23], [24] и [21].

2.1. Зайфертово разложение диаграммы зацепления и толстые графы. Несмотря на свое название, полиномы узлов/зацеплений на самом деле связаны не столько с зацеплениями и узлами, сколько с графами - и это подчеркивает, что наша история тесно связана с решеточными теориями. Основное отличие состоит в том, что данные графы - это графы достаточно общего типа, намного более 
широкого, нежели графы, связанные с регулярными периодическими решетками. Возможность их интерпретации в терминах узлов и зацеплений обусловлена инвариантностями Рейдеймейстера, главная из которых - это соотношение Янга-Бакстера. Таким образом, существенными в данном случае являются так называемые интегрируемые решеточные теории. Напрямую связаны с зацеплениями ориентированные $(2,2)$-валентные графы, которые можно интерпретировать как диаграммы зацеплений $\mathcal{L}$ - проекции ориентированных контуров с трех на два измерения. Для зацеплений в $\mathbb{R}_{3}$ (обычных зацеплений) диаграммы зацепления планарны; непланарные диаграммы связаны с виртуальными зацеплениями, т. е. зацеплениями, находящимися в неодносвязных пространствах-временах размерности $3 d$. Как следует из их интерпретаций как проекций, диаграммы зацеплений могут иметь вершины двух типов (цветов) - черные и белые.

В формализме Решетихина-Тураева (РT) [25]-[27] цвета связаны с $\mathcal{R}$-матрицей и обратной к ней, и в оригинальной версии [25] этого алгоритма существен также выбор направления на плоскости: после этого в точках поворота вставляются "поворачивающие матрицы" $q^{ \pm \rho}$. Однако эти вставки скорее связаны с циклами Зайферта и учитываются с помощью правильно определенных следов (или размерностей в контексте данной работы), так что выделенные направления не существенны в современных версиях формализма РТ. Что касается циклов Зайферта, то они появляются при разрешении каждой из вершин диаграммы $\mathcal{L}$ следующим образом:

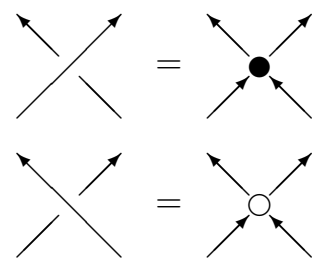

разрешение Зайферта

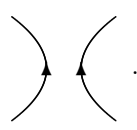

Тогда граф валентности $(2,2)$ превращается в набор из $\nu^{\mathcal{L}}$ непересекающихся ориентированных замкнутых линий, которые мы называем циклами Зайферта. На рис. 1 изображены диаграммы зацеплений с двумя компонентами (виртуальным зацеплением сверху и обычным снизу), которые разделяются соответственно на два и три цикла Зайферта, также показанных на рис. 1. Заметим, что согласно нашему определению разрешение Зайферта не зависит от раскраски вершин, а виртуальные (стерильные) пересечения вообще не разрешаются, т. е. для непланарной (виртуальной) диаграммы циклы Зайферта также непланарны. На рис. 1 и далее мы обозначаем виртуальные пересечения квадратами.

Следуя работе [21], в третьем столбце мы превращаем разрешенную по Зайферту диаграмму $\widehat{S}\left(\mathcal{L}_{c}\right)$ в граф $\Gamma^{\mathcal{L}_{c}}$, в котором $\nu^{\mathcal{L}}$ циклов Зайферта представлены вершинами, а исходные $n^{\mathcal{L}}$ пересечений - ребрами. Для сохранения информации о $\mathcal{L}$, мы сохраняем порядок, в котором ребра соединяются с вершинами, т. е. Г - это на самом деле толстый граф (детский рисунок). Во втором столбце на самом деле можно заменить точки в разрешенных вершинах на полосы - ребра толстого графа Г.

Другими словами, черные и белые вершины первого столбца, они же черные и белые точки в разрешенных вершинах во втором столбце, становятся соответственно пунктирными и сплошными жирными линиями в третьем столбце. Для сохранения непрерывности стрелок эти полосы на самом деле содержат переворот, как ленты 


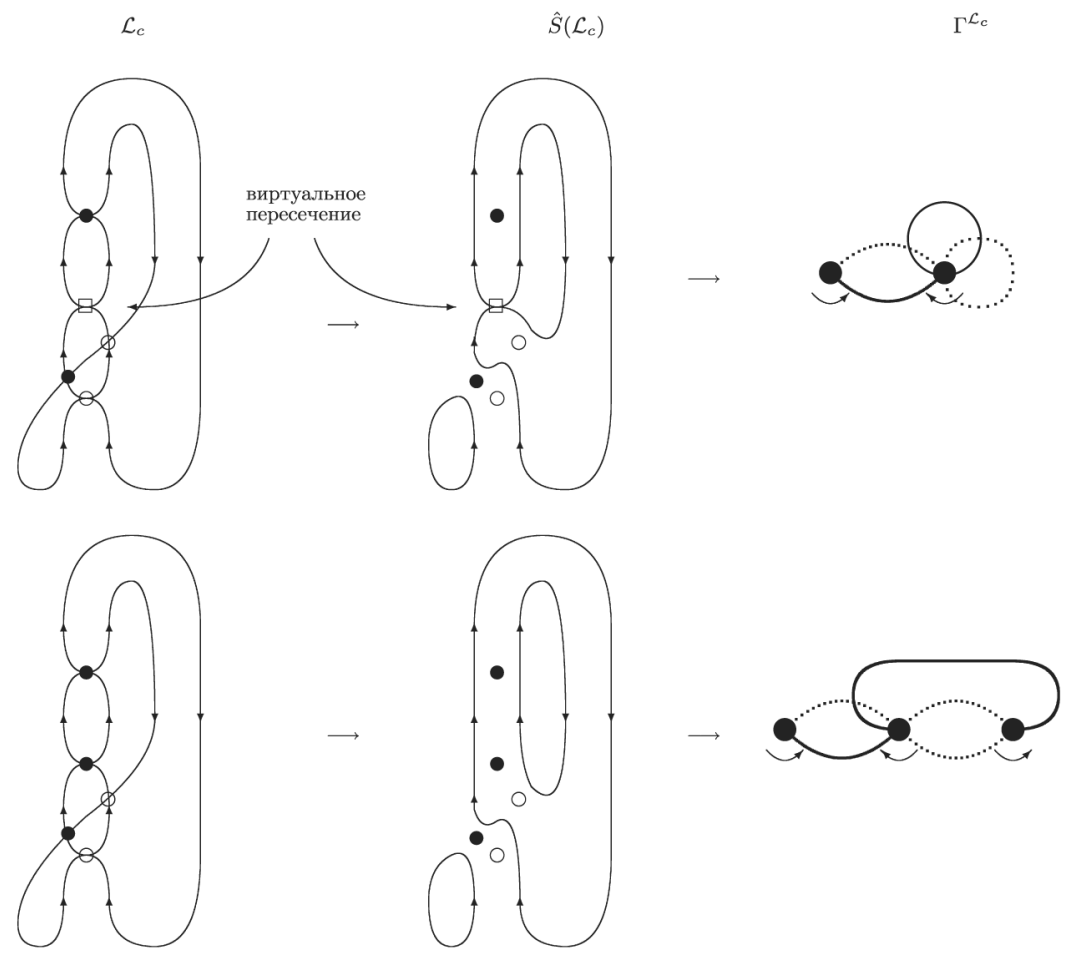

Рис. 1. Циклы Зайферта.

Мёбиуса, что является специфическим свойством соответствующей матричной модели (см. работу [21] и раздел 3 ниже). На рисунках можно заменить перекрутку ориентацией вершин, но это не работает в более сложных случаях. Толстые графы Г вновь появятся ниже в уравнении (6) и впоследствии будут играть существенную роль.

Альтернативное (или, возможно, параллельное) появление толстых графов в теории узлов описано в статье [28].

2.2. Раскраски и гиперкуб. Обозначим диаграммы зацеплений с раскрашенными вершинами как $\mathcal{L}_{c}$ - диаграмма $\mathcal{L}$ с $n^{\mathcal{L}}$ вершинами может иметь $2^{n}$ различных раскрасок $c$ (здесь и далее величины, связанные с диаграммой зацепления, обозначены верхним индексом $\mathcal{L}$, иногда мы будем опускать этот индекс для лучшей читаемости формул). Также можно рассмотреть два различных разрешения вершин - это опять-таки дает $2^{n}$ вариантов разрешения/разложения диаграммы $\mathcal{L}$. С диаграммой $\mathcal{L}$ мы связываем $n^{\mathcal{L}}$-мерный гиперкуб $\mathcal{H}^{\mathcal{L}}$, у которго $2^{n}$ вершин соответствуют различным разрешениям, а $2^{n-1} n$ ребер - переходам между двумя различными разрешениями определенной вершины диаграммы $\mathcal{L}$. Гиперкуб обладает выделенной зайфертовой вершиной $s$, соответствующей разрешению всех вершин в соответствии с $(1)$, и $\mathcal{L}$ при этом разделяется на $\nu^{\mathcal{L}}$ циклов Зайферта. Противоположная вершина $\bar{s}$ гиперкуба называется антизайфертовой. 
Заметим, что наш гиперкуб $\mathcal{H}^{\mathcal{L}}$ не зависит от раскраски $c$, его вершины описывают различные разрешения диаграммы $\mathcal{L}$. Раскраска $c$ связывается с определенной вершиной (которая называется начальной вершиной конкретного узла/зацепления) с помощью формального сопоставления двух раскрасок вершин с двумя разрешениями.

2.3. Разрешения и размерности. Идея формализма гиперкуба состоит в том, чтобы поставить в вершины гиперкуба некоторые объекты, определенные с помощью соответствующих разрешений, и связать морфизмы между этими объектами с изменениями разрешений вдоль ребер гиперкуба. Если эти морфизмы коммутируют, то гиперкуб превращается в абелев колчан, который далее может быть упрощен до комплекса, для которого можно построить полиномы Эйлера и Пуанкаре. Если вся эта конструкция удовлетворяет движениям Рейдемейстера, то эти величины можно интерпретировать как полиномы ХОМФЛИ и Хованова-Рожанского для узла/зацепления, который описывался диаграммой зацепления $\mathcal{L}_{c}$.

В исходной версии из работы [4] для обычных (невиртуальных) зацеплений и для группы $S L(2)$ (т. е. для $N=2$ ) эти объекты были просто $q$-градуированными векторными пространствами, а морфизмы определялись посредством операторов разрезания и склейки, связанных с переходами

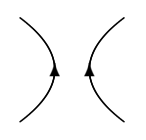

разрешение $S$ морфизмы разрезания и склейки

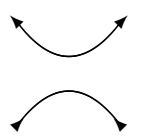

разрешение $K$

Каждая вершина гиперкуба $c \in \mathcal{H}^{\mathcal{L}}$ соответствует некоторой раскраске диаграммы $\mathcal{L}$. Если черные и белые вершины разрешаются как $S$ и $K$, то диаграмма разделяется на $\nu_{c}^{K}$ циклов, а соответствующее векторное пространство обладает квантовой ( $q$-градуированной) размерностью

$$
[N]^{\nu_{c}^{K}} \stackrel{N=2}{=}[2]^{\nu_{c}^{K}}, \quad \text { где } \quad[N]=\frac{q^{N}-q^{-N}}{q-q^{-1}}=\frac{\left\{q^{N}\right\}}{\{q\}}
$$

$([N]$ - это квантовое число $N)$. Здесь морфизмы суть линейные преобразования градуировки -1 , которые легко могут быть построены в явном виде, приводя (при $N=2$ ) к инвариантным относительно движений Рейдемейстера полиномам Джонса и Хованова.

Однако из рис. 1 очевидно, что разрешение $K$ не сохраняет стрелки, и, таким образом, может быть использовано только при $N=2$, когда направление не имеет значения. Для произвольного $N$ предложение, высказанное в работе [20], состоит в замене $K$ линейной комбинацией:

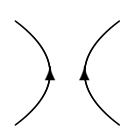

морфизмы разрезания и склейки

разрешение $S$

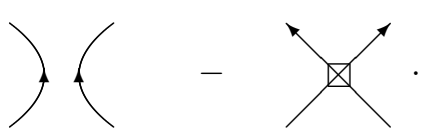

разрешение $D$ 
Уже имеется некоторый объем данных, свидетельствующий о том, что соответствующие ответы действительно являются инвариантными относительно движений Рейдемейстера полиномами ХОМФЛИ и Хованова-Рожанского. К сожалению, полное описание в данном случае не настолько прямолинейно, так как по сравнению со случаем $N=2$ присутствует ряд технических сложностей, которые тем не менее кажутся концептуально важными и неизбежными.

Во-первых, только для зайфертовой вершины $v=s$ размерность остается мономиальной, $d_{s}^{\mathcal{L}}=[N]^{\nu^{\mathcal{L}}}$. Однако, когда необходимо также разрешение второго типа (разрешение $D)$, размерность $d_{v}^{\mathcal{L}}$ полиномиальна по $N$ даже при $q=1$.

Во-вторых, для виртуальных узлов и зацеплений данные полиномы не обязательно положительны, и их отрицательные значения требуют интерпретации в терминах К-теории.

В-третьих, связанные с вершинами пространства в этом случае схожи с факторпространствами, с более сложными квантованием (градуировкой) и морфизмами, нежели в случае бесструктурных градуированных векторных пространств.

Все эти пункты делают описание размерностей и морфизмов сложной задачей, и настоящая работа является следующей ступенью на пути ее разрешения.

2.4. Полиномы ХОМФЛИ. Согласно работе [20] полиномы ХОМФЛИ в фундаментальном представлении представляются суммой

$$
H_{\square}^{\mathcal{L}_{c}}=\mathcal{C}^{\mathcal{L}_{c}} \sum_{v \in \mathcal{H}^{\mathcal{L}}}^{2^{n}}(-q)^{h(v, c)} \cdot d_{v}^{\mathcal{L}}(q, N)
$$

по всем вершинам $v$ гиперкуба $\mathcal{H}^{\mathcal{L}}$. В то время как гиперкуб и размерности зависят только от нераскрашенной диаграммы зацепления $\mathcal{L}$, полином ХОМФЛИ зависит и от раскраски, но в (2) эта зависимость проявляется только посредством весовых множителей $(-q)^{h(v, c)}$, где показатель степени равен числу ребер гиперкуба на кратчайшем пути от начальной вершины $c$ до $v$, и это единственный нетривиальный элемент в правой части, зависящий от $c$. В топологическом оснащении также присутствует общий зависящий от раскраски множитель перед суммой по $v$ :

$$
\mathcal{C}^{\mathcal{L}_{c}}=q^{(N-1) n \bullet} \cdot\left(-q^{-N}\right)^{n_{\circ}},
$$

где $n_{\bullet}$ и $n_{\text {。 }}$ - полное число черных и белых вершин на диаграмме зацепления $\mathcal{L}_{c}$.

При $q=1$ явная форма разрешения $D$ подразумевает, что размерность

$$
\left.d_{v}^{\mathcal{L}}\right|_{q=1}=\sum_{s \leqslant w \leqslant v}(-1)^{h(w, s)} N^{\nu_{w}^{\times}}
$$

равна сумме по всем вершинам подкуба $\mathcal{H}^{\mathcal{L}}$, расположенного между вершиной $v$ и зайфертовой вершиной $s$; здесь $\nu_{w}^{\times}-$это число циклов, появляющихся, если разрешить все белые пересечения для раскраски $w$ посредством соотношения

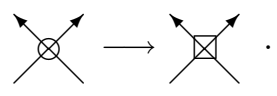


Заметим, что $\nu_{w}^{\times} \neq \nu_{w}^{K}$, и в этой формуле нет ничего похожего на число циклов $\nu_{w}^{D}$, связанного с составным разрешением $D$ белых вершин раскраски $w$. После подстановки равенства (4) выражение (2) становится двойной суммой:

$$
\begin{aligned}
\left.H_{\square}^{\mathcal{L}_{c}}\right|_{q=1} & =\sum_{v \in \mathcal{H}^{\mathcal{L}}}(-1)^{h(v, c)} \sum_{s \leqslant w \leqslant v}(-1)^{h(w, s)} N^{\nu_{w}^{\times}}= \\
& =\sum_{w \in \mathcal{H}^{\mathcal{L}}}(-1)^{h(w, s)} N^{\nu_{w}^{\times}} \sum_{w \leqslant v \leqslant \bar{s}}(-1)^{h(v, c)}=(-1)^{h(\bar{s}, c)+h(\bar{s}, s)} N^{\nu_{\bar{s}}^{\times}} .
\end{aligned}
$$

Так как сумма по $v$ не равна нулю только при $w=\bar{s}$, двойная сумма сводится к одному слагаемому в антизайфертовой вершине, где $\nu_{\bar{s}}^{\times}=l^{\mathcal{L}}$ равно количеству компонент зацепления $\mathcal{L}$. Однако при $q \neq 1$ эти две суммы квантуются ( $q$-деформируются) совершенно разным образом, сокращения намного менее явные, и двойная сумма дает полином ХОМФЛИ, являющийся нетривиальным квантованием простого случая $N^{l}$. Ключевой момент, естественно, состоит в квантовании размерностей (4).

2.5. Квантование размерности $d_{v}^{\mathcal{L}}$. Существует несколько уровней понимания данной процедуры.

Во-первых [20], в простейшем случае суммы (4) дают полиномы по $N$ с целыми корнями, т. е. разлагаются в произведение мономов вида $N-k$. Такие произведения естественным образом $q$-деформируются в аналогичное произведение квантовых чисел $[N-k]$.

Во-вторых [20], можно использовать тот факт, что размерности не зависят от раскраски. Одна и та же диаграмма $\mathcal{L}$ при различных раскрасках может описывать как сложные, так и довольно простые узлы/зацепления, иногда настолько простые, как отсутствие узла (неузел). Если предполагать топологическую инвариантность соотношения (2), то эта идея дает рекурсивную процедуру для вычисления более сложных размерностей через более простые.

В-третьих [23], можно вывести размерности из формализма РТ [25]-[27], где ответ дается следами комбинаций проекторов $P_{[11]}$ на антисимметрическое представление в произведении двух фундаментальных представлений. Несмотря на то что априори не существует причин, по которым этот подход может быть применен к виртуальным узлам, он вполне применим и для них. Более того, полученные результаты предполагают, что теория представлений не согласована с виртуальными пересечениями только в симметрическом канале, тогда как в асимметрическом проблем не возникает (см. впечатляющие примеры в работе [24]).

В-четвертых [21], классические размерности (4) зависят только от подкуба между вершинами $v$ и $s$, но не от всего гиперкуба и, таким образом, не от всей диаграммы $\mathcal{L}$; квантование сохраняет это свойство. Формализм, который учитывает это свойство и позволяет должным образом разделить переменные, использует толстые графы $\Gamma^{\mathcal{L}_{c}}$, введеные выше в п. 2.1. Вдобавок в рамках этого формализма вводятся матрично-модельное представление и свойства, что позволяет систематизировать различные рекурсивные идеи и наблюдения. 
2.6. Полиномы ХОМФЛИ, выраженные через толстые графы. В работе [21] мы предложили преобразовать выражение (2) в сумму по всем $2^{n^{\mathcal{L}}}$ подграфам $\bar{\gamma}$ толстого графа $\Gamma^{\mathcal{L}_{c}}$ с $n^{\mathcal{L}}$ ребрами:

$$
H_{\square}^{\mathcal{L}_{c}}=\mathcal{C}^{\mathcal{L}_{c}} \sum_{\bar{\gamma} \subset \Gamma^{\mathcal{L}_{c}}}(-q)^{\# \text { обращений }} \cdot D_{\bar{\gamma}}(q, N),
$$

где суммирование ведется по всем подграфам $\bar{\gamma}$ в обращенном графе $\Gamma^{\mathcal{L}_{c}}$. Напомним, что, как было сказано в п. 2.1, вершины графа $\Gamma^{\mathcal{L}_{c}}$ соответствуют $\nu^{\mathcal{L}}$ зайфертовым циклам в диаграмме $\mathcal{L}$, тогда как $n^{\mathcal{L}}$ ребер связаны с невиртуальными пересечениями в диаграмме $\mathcal{L}$. Раскраска $c$ превращает некоторые из ребер в пунктирные. Сумма в (6) производится по всем изменениям раскрасок: замене сплошных ребер на пунктирные и наоборот - пунктирных на сплошные. Размерности, однако, не “знают” о существовании пунктирных линий:

$$
D_{\bar{\gamma}}=D_{\bar{\gamma}_{\mathrm{amp}}}
$$

где $\bar{\gamma}_{\text {amp }}$ - это граф $\bar{\gamma}$, в котором все пунктирные линии стерли (обрезали).

Элементарное комбинаторное упражнение показывает, что сумма по изменениям раскрасок может быть переписана как несколько другая сумма по обрезанию ребер:

$$
H_{\square}^{\mathcal{L}_{c}}=q^{(N-1)\left(n_{\ldots}\left(\Gamma^{\mathcal{L}_{c}}\right)-n_{-}\left(\Gamma^{\mathcal{L}_{c}}\right)\right)} \sum_{\gamma \subseteq \Gamma^{\mathcal{L}_{c}}}(-q)^{n_{\ldots}(\gamma)-n_{-}(\gamma)} \cdot D_{\gamma}(q, N),
$$

где $n_{-}(\gamma)$ и $n_{\ldots}(\gamma)$ обозначают число сплошных и пунктирных линий в графе $\gamma$. По аналогии с $\mathcal{C}^{\mathcal{L}_{c}}$ показатель степени в префакторе этого выражения - это просто антисимметризация числа сплошных и пунктирных линий, $n_{-}\left(\Gamma^{\mathcal{L}_{c}}\right)=n_{\bullet}\left(\Gamma^{\mathcal{L}_{c}}\right)$ и $n_{\ldots}\left(\Gamma^{\mathcal{L}_{c}}\right)=n_{\circ}\left(\Gamma^{\mathcal{L}_{c}}\right)$. В этом случае суммирование ведется по всем подграфам $\gamma$ графа $\Gamma^{\mathcal{L}_{c}}$ с тем же набором вершин, но с обрезанием некоторых ребер, тогда как тип оставшихся ребер сохраняется и входит в формулы посредством $n_{-}(\gamma)$ и $n_{\ldots}(\gamma)$. Таким образом, вместо того чтобы вообе не зависеть от пунктирных линий, размерности $D_{\gamma}$ зависят от них так же, как от сплошных:

$$
D_{\gamma}=D_{\gamma_{\text {colorless }}},
$$

где $\gamma_{\text {colorless }}$ - это граф $\gamma$, про раскраску ребер которого мы забыли.

Такая кажущаяся безвредной замена суммирования и замена обозначений имеет важные последствия: вклад конкретного подграфа $\gamma$ в полином ХОМФЛИ, т. е. как $(-q)$-префактор, так и квантовая размерность, полностью определяется самим $\gamma$, но не исходным $\Gamma^{\mathcal{L}_{c}}$. Если один и тот же граф $\gamma$ возникает в суммах для двух различных диаграмм зацеплений $\mathcal{L}_{1}$ и $\mathcal{L}_{2}$, то в обоих случаях вклады от этого графа в точности равны друг другу. Это не так в исходном выражении (6), где квантовые размерности одинаковы, но $(-q)$-множители могут отличаться и зависят от раскраски ребер.

ПримеР. Зайфертова вершина для торического узла $(2,4)$. Сумма по изменению раскрасок имеет вид

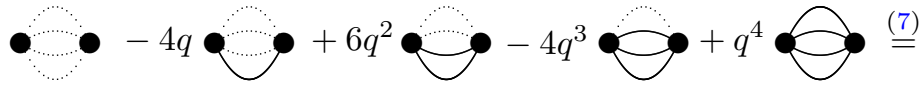

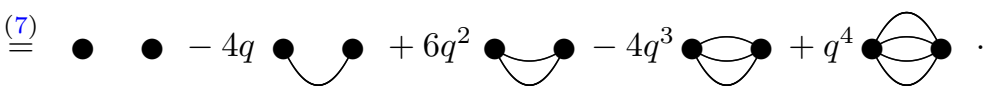


Сумма по обрезанию ребер (для всех черных вершин она идентична сумме по изменению раскрасок) имеет вид

$$
\begin{aligned}
& q^{4} \bullet-4 q^{3} \bullet \bullet+6 q^{2} \bullet \bullet-4 q \bullet \bullet+\bullet \bullet \stackrel{(9)}{=} \\
& \stackrel{(9)}{=} q^{4} \circlearrowleft-4 q^{3} \leqq+6 q^{2} \bigcirc-4 q \circlearrowleft
\end{aligned}
$$

Пример. Незайфертова вершина торического узла $(2,4)$. Сумма по изменению раскрасок (слагаемые, которые находятся на одной вертикали, имеют одинаковые $(-q)$-префакторы) такова:

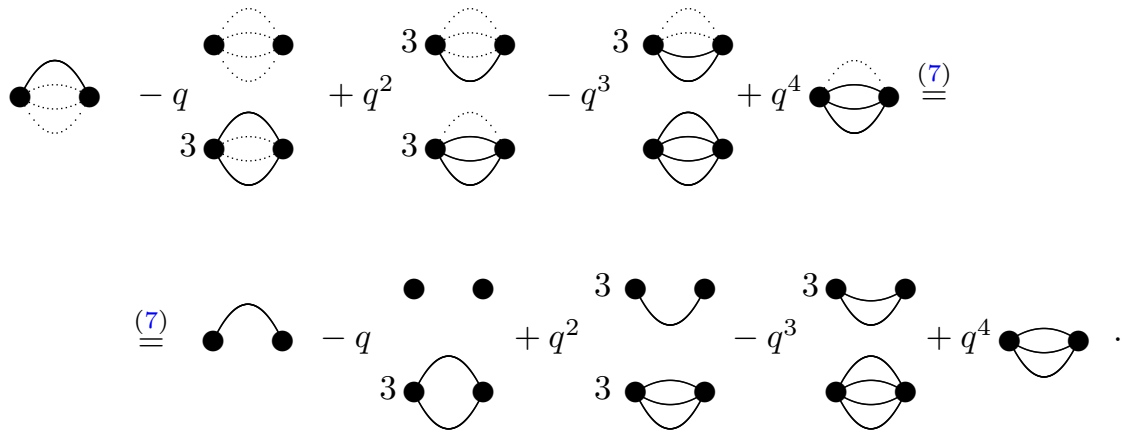

Сумма по обрезанию ребер (находящиеся на одной вертикали слагаемые имеют одинаковое число стертых ребер) имеет вид

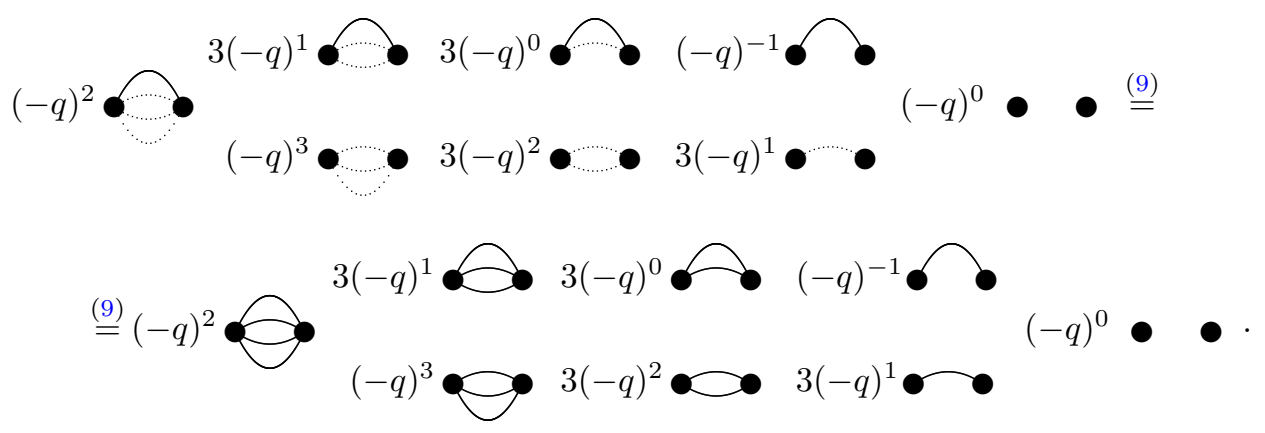

Дополнительный множитель $-1 / q$ в суммировании по обрезанию ребер компенсируется разницей между $\mathcal{C}^{\mathcal{L}_{c}}$ и $q^{(N-1)\left(n_{\ldots} . n_{-}\right)}$.

В такой версии формализма толстого графа/матричной модели считается заданным, что квантовые размерности $D_{\gamma}$ и $q$-веса $(-q)^{n_{\ldots}-n_{-}}$зависят только от $\gamma$, который сам по себе является толстым графом, но не зависят ни от $\mathcal{L}_{c}$, ни даже от $\Gamma^{\mathcal{L}_{c}}$, т. е. что конструкция гиперкуба для полинома ХОМФЛИ локальна. Это свойство локальности, которое в данном тексте критично для доказательства инвариантности относительно движений Рейдемейстера, может сохраниться или нет при переходе к суперполиномам (полиномам Хованова-Рожанского). По крайней мере 
наивно, конструкция Хованова-Рожанского нелокальна, так как целые слои вершин (в отличие от отдельных вершин) гиперкуба явно входят в ее формулировку. Таким образом, переопределение конструкции Хованова-Рожанского в локальных терминах представляется важным направлением будущих исследований.

Две формулы суммирований (2) и (8) тесно связаны друг с другом, и квантовые размерности $D_{\gamma}$ и $d_{v}$ по сути представляют собой одни и те же величины. Однако соотношение (8) позволяет подчеркнуть особые свойства квантовых размерностей, например быструю редукцию, которые не так просто выразить с помощью равенства (2). Это связано с тем, что $D_{\gamma}$ и $d_{v}$ - функции разных переменных. Свойства такого типа дают формализму гиперкуба дополнительный слой локальности (размерности $D_{\gamma}$ изменяются хорошо при локальных преобразованиях толстого графа). Это, вместе с упомянутой выше локальностью вершин, критично для инвариантности относительно движений Рейдемейстера. Более того, присутствуют намеки (см. раздел (7) на то, что инвариантность относительно движений Рейдемейстера - это только вершина айсберга, связанного с локальностью, и на самом деле намного более богатые структуры (схожие, а, возможно, и в точности совпадающие, с топологической рекурсией АMМ/ЭО [13]) скрываются в глубине. Это, конечно, ожидаемо с точки зрения матричных моделей.

2.7. Свойства факторизации. Размерности удовлетворяют свойству факторизации: для разделенного графа

$$
D_{\gamma}=D_{\gamma_{1}} \cdot D_{\gamma_{2}}, \quad \text { если } \quad \gamma=\gamma_{1} \cup \gamma_{2} \quad \text { при } \quad \gamma_{1} \cap \gamma_{2}=\varnothing \text {, }
$$

что в точности совпадает со свойством, выполнение которого ожидалось бы при вычислениях через след.

Более того, существует также более сильное свойство факторизации: если граф состоит из двух частей, связанных посредством одной вершины, и ребра этих графов не переплетены, то нормированные размерности $D(\gamma) /[N]$ также факторизуются:

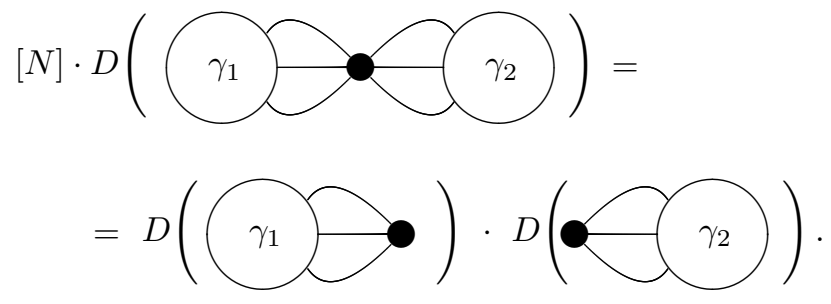

Вкупе с явным выражением для

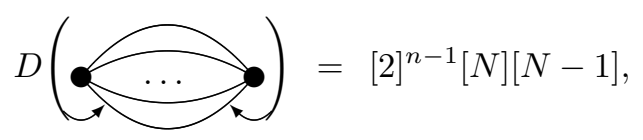

где $n \geqslant 1$ - это число ребер на данной простой толстой диаграмме (для упрощения рисунка вершины нарисованы противонаправленными, и потому диаграмма не 
перекручена), возможно обобщение

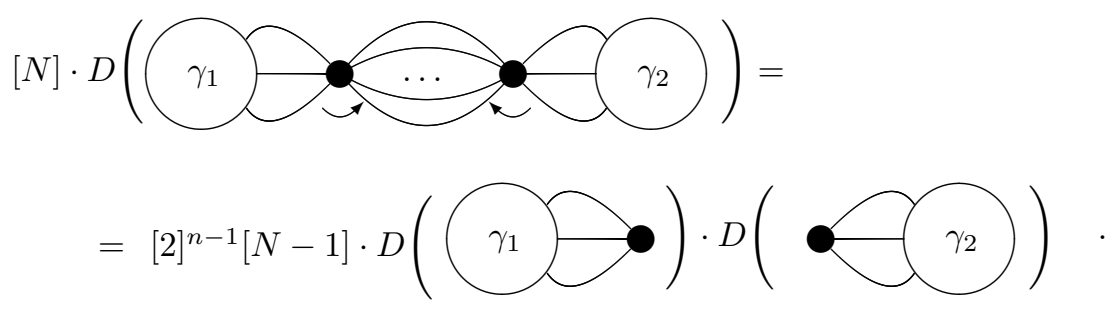

Выражение (11) можно считать следствием того, что нормированные полиномы ХОМФЛИ составных узлов (как обычных, так и виртуальных) можно представить как произведение нормированных полиномов ХОМФЛИ их составляющих. Достаточно отметить, что толстый граф составных узлов в точности имеет вид правой части равенства (11); по этой причине ниже мы будем называть такие графы составными.

ЗАмечАниЕ 1. Вместе выражения (10) и (11) фиксируют, что размерность отдельной вершины равна $[N]$, так как

$$
\text { • } \stackrel{(11)}{=} \frac{1}{[N]} \bullet \bullet \stackrel{(10)}{=} \frac{1}{[N]} \bullet^{2} \longrightarrow \bullet=[N] \text {. }
$$

2.8. Резюме. Оставшаяся задача в части, затрагивающей полиномы ХОМФЛИ обычных и виртуальных зацеплений и узлов, разделяется на три практические задачи:

- построение толстых графов для конкретных диаграмм узлов;

- вычисление квантовых размерностей $D_{\gamma}$ для различных (в идеале произвольных) толстых графов $\gamma$;

- подтверждение того, что ответы для полиномов ХОМФЛИ инвариантны относительно движений Рейдемейстера.

Алгоритм решения первой задачи должен быть ясен из примеров в п. 2.1. Дополнительные свойства, такие как инъективность и сюръективность этого преобразования, и дополнительные примеры приведены в приложении А. Вторую задачу мы рассмотрим в деталях в разделе 3 , заострив внимание на связи с матрично-модельными корреляторами. Затем в разделе 4 мы используем матрично-модельные интуитивные представления для поиска рекурсивных соотношений и их обобщений на случай $q \neq 1$. При этом легко получаются соотношения, отражающие инвариантность относительно движений Рейдемейстера (см. раздел 5). Несмотря на то что данные соотношения не являются в полной мере рекурсией, добавление всего лишь одного дополнительного соотношения (“флипа") достаточно для построения эффективной компьютерной программы (см. раздел 6), которая рассчитывает размерности $D_{\gamma}$ и полиномы ХОМФЛИ с помощью подхода гиперкуба для всей таблицы Рольфсена $^{1)}$. Последний раздел 7 посвящен поиску более продвинутых рекурсий, которые могут расширить возможности компьютерных вычислений. Помимо других аспектов, это включает в себя расширение на диаграммы валентности более высокой, чем

1) CM. http://katlas.org/wiki/The_Rolfsen_Knot_Table, http://www . math. toronto. edu/drorbn/Students/GreenJ/. 
$(2,2)$, что, как предсказывалось в работе [20], открывает новые горизонты для всей теории.

Важный шаг вперед по сравнению с работой [21] состоит не только в более детальном, полном и расширенном описании, но и в доказательстве того, что выражение (8) обладает инвариантностью относительно движений Рейдемейстера. Что касается взаимодействия между диаграммами зацеплений (возможно, и обобщенных на другие валентности) и толстых графов, загадки еще остаются: на данный момент некоторые вещи удобнее формулировать на одном языке, а другие - на другом. Это не такая редкость, когда задействованы дуальные описания, однако лучшее понимание и адекватное переформулирование появляющихся рекурсий, конечно, необходимы.

\section{3. МАТРИЧНО-МОДЕЛЬНЫЕ КОРРЕЛЯТОРЫ И ИХ $q$-ДЕФОРМАЦИЯ}

Начнем данный раздел с разложений по родам с нормальным упорядочением и без нормального упорядочения для корреляторов

$$
\left\langle\prod_{i=1}^{m} \operatorname{Tr} M^{k_{i}}\right\rangle .
$$

Эти разложения получаются с помощью правила Вика из пропагатора

$$
\pi_{j l}^{i k}=\left\langle M_{j}^{i} M_{l}^{k}\right\rangle^{(0)}=\delta_{l}^{i} \delta_{j}^{k}
$$

при этом различные члены разложения (конкретные графы/диаграммы Фейнмана) дают вклад в различные зацепления (в общем случае виртуальные). Это задает преобразования зацепление $\mathcal{L} \longrightarrow$ толстый граф $\Gamma^{\mathcal{L}}$.

После этого классические $(q=1)$ размерности $d_{v}^{\mathcal{L}}$ в вершинах гиперкуба $\mathcal{H}^{\mathcal{L}}$ можно получить с помощью замены в ребрах графа $\Gamma^{\mathcal{L}}$ пропагатора (15) на пропагатор

$$
\Pi_{j l}^{i k}=\delta_{j}^{i} \delta_{l}^{k}-\delta_{l}^{i} \delta_{j}^{k},
$$

что дает выражения для диаграмм Фейнмана в виде линейной комбинации подграфов графа $\Gamma^{\mathcal{L}}$.

Получившиеся комбинации далее должны быть проквантованы ( $q$-деформированы), и это самая сложная часть истории. Квантование работает по правилам из работы [23], исходно пришедшим из подхода РТ [25]-[27], обобщенным на виртуальный случай в статье [24]. Следуя предложению, сделанному в работе [21], мы переформулируем эти правила в терминах соотношений матрично-модельной рекурсии: основываясь на идее, что некоторые из рекурсий, связанные с движениями Рейдемейстера, должны сохраниться после квантования. Таким образом, мы получаем универсальный метод вычисления, применимый не только к обычным, но и к виртуальным узлам, для которых прямое использование метода РТ невозможно. Что более важно, мы получаем легко компьютеризируемый формализм, с помощью которого можно получить многие (если не все) из необходимых размерностей (в [21] он был применен к диаграммам $\mathcal{L}$ с 14 пересечениями и менее, т. е. к гиперкубам $\mathcal{H}^{\mathcal{L}}$ с не более чем $2^{14}$ вершинами). 
Цель данного раздела состоит в том, чтобы перечислить начальные из списка размерностей (большее число примеров приведено в дополнении В), тогда как происхождение и значение этого списка будут обсуждаться в последующих разделах. Мы приводим только самые сложные размерности, связанные с антизайфертовой вершиной $\bar{S}$ гиперкуба (см. табл. 1, 2). Размерности в других вершинах соответствуют подграфам $\Gamma^{\mathcal{L}}$ с обрезанными ребрами, которые приведены в более высоких строках таблиц. Когда граф состоит из нескольких отдельных компонент, размерности факторизуются в соответствии с (10). Даже эти короткие таблицы могут казаться пугающими. Однако отметим несколько важных моментов, отраженных в них.

Во-первых, ответы (размерности) намного менее разнообразны, чем сами толстые графы: с точностью до общего знака все отличные друг от друга ответы возникают уже при рассмотрении одноточечного коррелятора. Это является экспериментальным доказательством правила флипа, описанного в следующем разделе.

Во-вторых, ответы для раздельных графов равны произведению ответов для компонент, и это свидетельствует о наличии свойства факторизации (10).

В-третьих, толстые графы, помеченные звездочкой в последнем столбце таблиц, нарушают наивное правило квантования: тогда как классическая размерность содержит простой линейный множитель $N-3$, его квантование $[N-2]-1$ нетривиально, и это свидетельствует о том, что вся история не сводится просто к неким правилам квантования на уровне полиномов: нужен какой-то формализм типа формализма толстых графов.

В-четвертых, различные виртуальные узлы часто соответствуют одним и тем же толстым графам (см. также пример знаменитого узла Кишино в приложении А).

Наконец, столбец $\mathcal{L}$ пуст для некоторых толстых графов. Это связано не с тем, что не существует соответствующих диаграмм зацеплений, но с тем, что нам не известны достаточно простые диаграммы зацеплений, которые давали бы такие толстые графы. В приложении А описан метод построения (довольно сложных) диаграмм зацеплений для произвольного толстого графа.

Более формально, произвольный матрично-модельный коррелятор разлагается в сумму графов Фейнмана $\langle\Gamma\rangle$, связанных с толстыми графами $Г$ : мы имеем теорему Вика

$$
\left\langle\prod_{i} \operatorname{Tr} M^{k_{i}}\right\rangle=\sum_{\Gamma} C_{\Gamma}\left\{k_{i}\right\} \cdot\langle\Gamma\rangle .
$$

Из-за особой формы пропагатора (16) каждый граф

$$
\langle\Gamma\rangle=\left.\sum_{\gamma \subset \Gamma}(-1)^{E_{\Gamma}-E_{\gamma}} D_{\gamma}(q)\right|_{q=1}
$$

равен сумме с чередующимися знаками по всем толстым подграфам $\gamma$ графа $Г$, где вклад графа $\gamma$ равен классической размерности $D_{\gamma}(q)$ при $q=1$.

Толстый граф $Г$ можно построить для произвольной диаграммы зацепления $\mathcal{L}$ (хотя разные диаграммы зацеплений могут соответствовать одному и тому же графу - преобразование $\mathcal{L} \rightarrow \Gamma$ на самом деле сюръективно, а не инъективно, см. приложение А), и это дает все составляющие выражения (8) для полиномов ХОМФЛИ при $q=1$. 


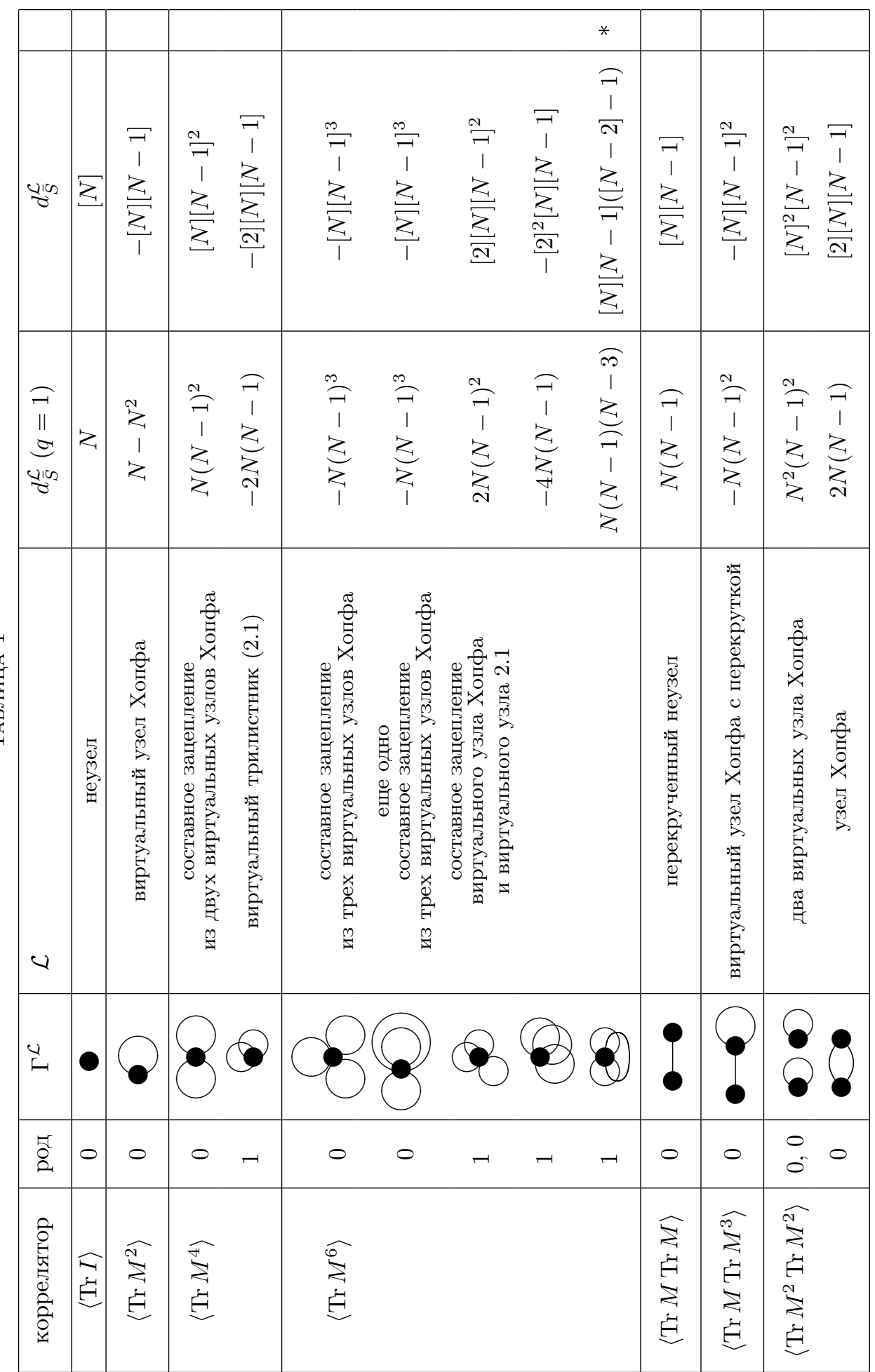




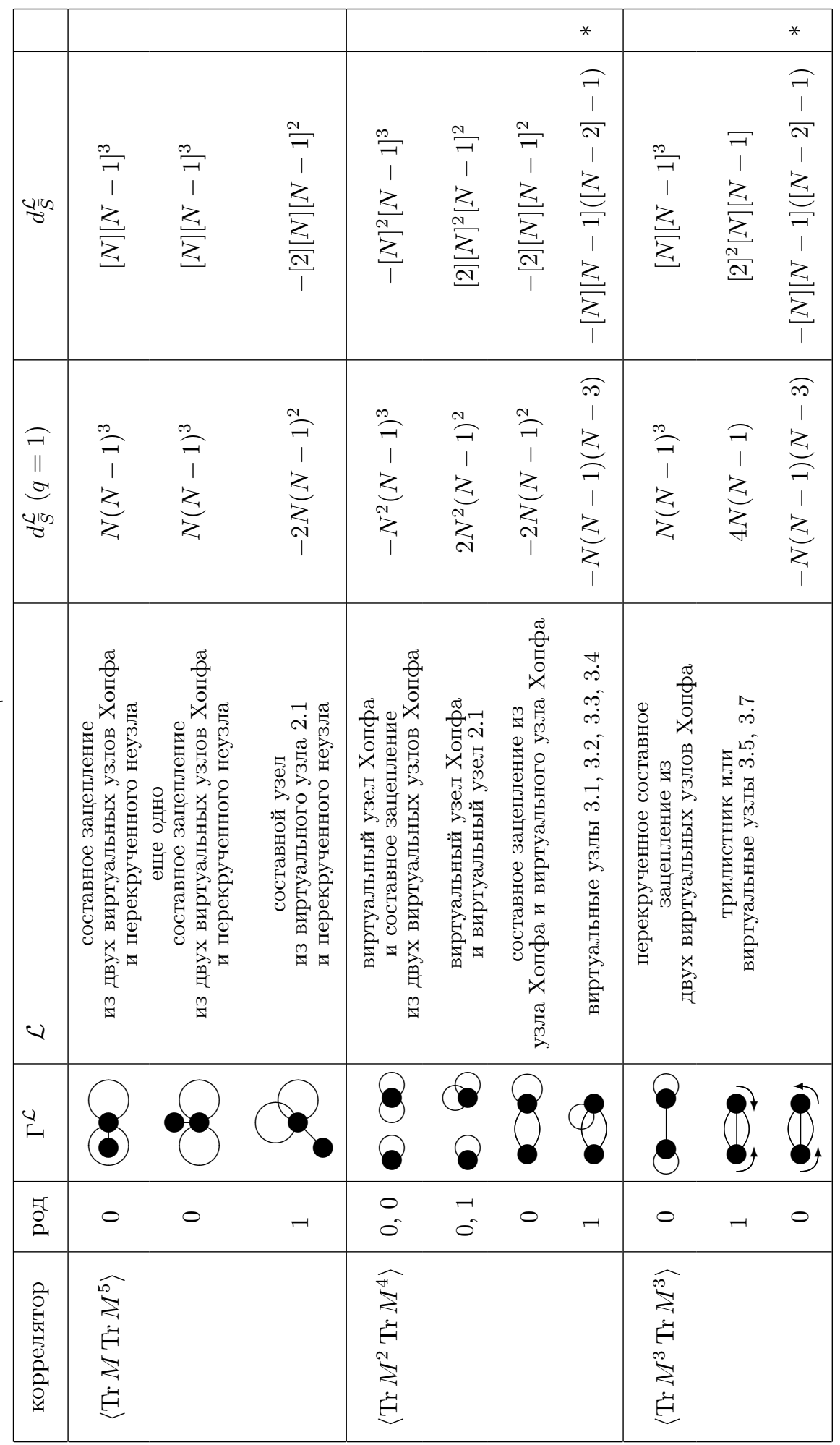


Полученный результат приводит к следующим трем предметам нашего обсуждения в оставшейся части работы.

- Матрично-модельные соотношения Уорда для корреляторов $\left\langle\prod_{i} \operatorname{Tr} M^{k_{i}}\right\rangle$ задают рекурсивные соотношения для классических размерностей $D_{\gamma}(q)$ при $q=1$.

- Процедура квантования $\left.D_{\gamma}(q)\right|_{q=1} \longrightarrow D_{\gamma}(q)$.

- Возможность обобщения соотношений Уорда и само́й матричной модели на случай $q \neq 1$.

Как было сказано в работе [21], можно обобщить три соотношения Уорда, связанные с инвариантностью относительно движений Рейдемейстера. Это очень важно само по себе, так как эти соотношения локальны (т. е. верны всякий раз, когда имеется подходящий фрагмент толстого графа, вне зависимости от величины и сложности всего толстого графа), тогда как размерности сами по себе определены глобально (зависят от всего графа в целом). Тем не менее перевод этого ограниченного набора тождеств Уорда в рекурсию составляет отдельную задачу. Наша нынешняя компьютерная программа решает ее довольно грубо и неэффективно с полным перебором на некотором шаге (см. раздел 6, где мы также обсуждаем возможность более разумного подхода). Однако даже нынешний грубый подход позволяет воспроизвести фундаментальные полиномы ХОМФЛИ для всех узлов из таблицы Рольфсена. Для вычисления даже простейших примеров виртуальных узлов необходимо дополнительное преобразование флипа, которое странным образом выживает при $q \neq 1$. Заодно оно позволяет более эффективно вычислять полиномы ХОМФЛИ для невиртуальных узлов.

\section{4. ЛОКАЛЬНЫЕ ПРЕОБРАЗОВАНИЯ ТОЛСТОГО ГРАФА ПРИ $q \neq 1$}

Несмотря на то что теоретически квантовые размерности $D_{\gamma}$ для любого толстого графа $\gamma$ могут быть вычислены напрямую из подхода РТ [23] (и для некоторых узлов/зацеплений этот метод действительно крайне эффективен), на практике мы не пользуемся этим способом. Вместо этого, глядя на ответы, полученные в работе [23], мы вывели в [21] набор соотношений, которым, по-видимому, удовлетворяют $D_{\gamma}$. Это преобразования толстого графа $\gamma$, которые сохраняют связанные с ним квантовые размерности $D_{\gamma}$. Ключевой момент состоит в том, что эти преобразования локальны: они связаны только с маленькими подграфами (с одной, двумя и тремя вершинами) и остаются верными при произвольном сложном окружении, присоединенном с обеих сторон равенства. Другими словами, несмотря на то что размерности сами по себе зависят от всего графа (т. е. нелокальны), соотношения между ними возникают, когда изменяется всего лишь маленький подграф. На рисунке ниже толстые стрелки обозначают части вершин толстого графа, а тонкие линии - ребра толстого графа. Предполагается, что остальной граф не изменяется при этих преобразованиях, и он не показан на рисунках. Также отметим, что согласно обозначениям, принятым нами при рассмотрении суммирования по обрезанию ребер в п. 2.6, любое сплошное ребро можно заменить на пунктирное (это не влияет на квантовые размерности, а только на зависящий от $q$ префактор). 
Полученные нами соотношения чисто экспериментальные, но они обладают рядом свойств, которые в некотором смысле их подтверждают. Во-первых, конечно, эти соотношения верны при $q=1$, когда они являются элементарными следствиями явной формы пропагатора (16) и правила Вика. Во-вторых, вычисление размерностей $D_{\gamma}$ только с помощью этих соотношений и свойств факторизации (10) и (11) и подстановка их в выражение (8) дает правильные ответы для фундаментальных полиномов ХОМФЛИ для всех узлов из таблицы Рольфсена (описание алгоритма приведено в разделе 6). В-третьих, в следующем разделе мы покажем, что этих соотношений и свойств факторизации (10) и (11) достаточно для выполнения свойства инвариантности относительно движений Рейдемейстера.

Так или иначе, приведем эти соотношения (мы также приводим соответствующие соотношения при $q=1$, полученные из матрично-модельных корреляторов).

- Правило $[N-1]$ состоит в том, что можно свернуть все вершины валентности 1:

$$
\begin{gathered}
\left.C \sim[N-1]\right|^{4}, \\
\langle\operatorname{Tr} M F(M)\rangle=(N-1) \delta_{l}^{k}\left\langle\frac{\partial F}{\partial M_{l}^{k}}\right\rangle .
\end{gathered}
$$

- Правило [2] состоит в том, что можно сократить двойные ребра (в этом случае важна взаимная ориентация вершин толстого графа):

$$
\sum_{\left.\left\langle M_{j}^{i} M_{k}^{j} M_{m}^{l} M_{n}^{m} F_{i l}^{k n}(M)\right\rangle=[2]\right)}(
$$

- Правило $[N-2]$ состоит в том, что можно сократить все двухвалентные вершины:

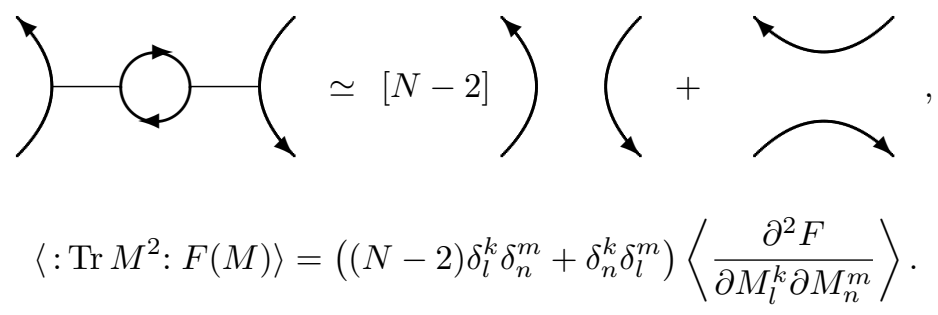


- Правило $[N-3]$ состоит в том, что можно подвергнуть "мутации" трехвалентные вершины:

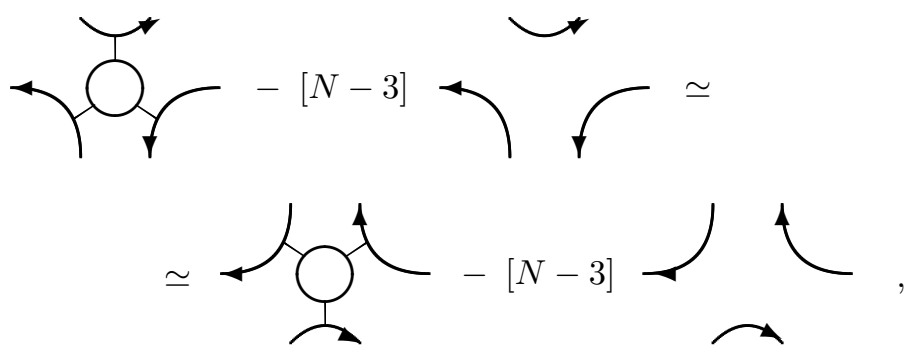

$$
\begin{aligned}
\left\langle: \operatorname{Tr} M^{3}: F(M)\right\rangle= & \left((N-3) \delta_{m}^{l} \delta_{p}^{n} \delta_{s}^{r}+\left(\delta_{m}^{n} \delta_{p}^{l} \delta_{s}^{r}+\delta_{m}^{l} \delta_{p}^{r} \delta_{s}^{n}+\delta_{p}^{n} \delta_{s}^{l} \delta_{m}^{r}\right)-\delta_{m}^{r} \delta_{p}^{l} \delta_{s}^{n}\right) \times \\
& \times\left\langle\frac{\partial^{3} F}{\partial M_{m}^{l} \partial M_{p}^{n} \partial M_{s}^{r}}\right\rangle .
\end{aligned}
$$

- Правило 1 состоит в том, что можно переставить ребра между тремя вершинами, как показано на рисунке:

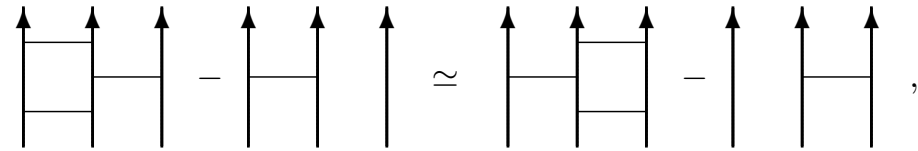

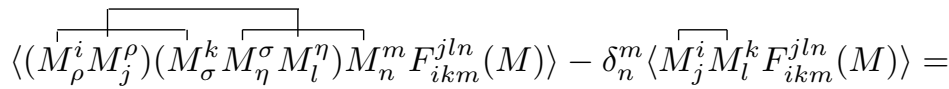

$$
\begin{aligned}
& \left.=\left\langle{ }_{M_{j}^{i}\left(M_{\sigma}^{k} M_{\eta}^{\sigma}\right.}^{\left.M_{l}^{\eta}\right)\left(M_{\rho}^{m}\right.} M_{n}^{\rho}\right) F_{i k m}^{j l n}(M)\right\rangle-\delta_{j}^{i}\left\langle{\overparen{M_{l}^{k}}}_{M_{n}^{m}}^{m} F_{i k m}^{j l n}(M)\right\rangle .
\end{aligned}
$$

- Правило флипа состоит в том, что можно изменить число вершин в толстом графе:

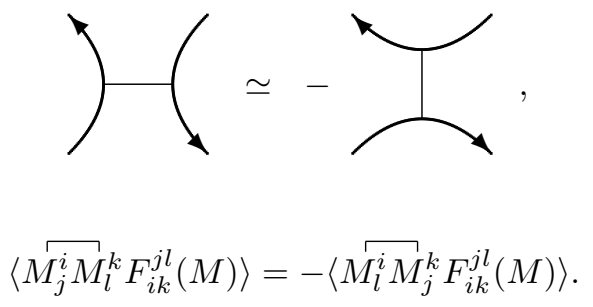

Заметим, что матрично-модельные аналоги преобразований (20), (23) и (24) несколько отличаются от своих аналогов (19), (21) и (22). Несмотря на то что при $q=1$ их одинаково просто вывести из явной формы пропагатора (16), их не так легко записать в терминах нормальных упорядочений. Это связано с тем, что знак нормального упорядочения позволяет легко понять, какие матрицы не следует спаривать, но нельзя сказать, какие матрицы следует спаривать. Описанные выше правила и являются этими "спаривающими" соотношениями, которые нужно описать алгебраически; таким образом мы ограничиваемся явным знаком спаривания. 
Как мы уже упоминали в работе [21], правило флипа (24) отличается от всех остальных правил из этого раздела. Как продемонстрировано в следующем разделе, оно не требуется для инвариантности относительно движений Рейдемейстера (как при обычных, так и при виртуальных движениях!). Поэтому в принципе должна иметься возможность определить размерности $D_{\gamma}$ каким-то другим образом, чтобы выполнялись все правила данного раздела, кроме правила флипа, а результаты вычисления с помощью метода гиперкуба (8) оставались топологически инвариантными. Изучение таких возможностей, однако, находится за пределами рассмотрения настоящей статьи.

\section{5. ПОДТВЕРЖДДЕНЕ РЕКУРСИВНЫХ ПРАВИЛ С ПОМОЩЬЮ ИНВАРИАНТНОСТИ ОТНОСИТЕЛЬНО ДВИЖЕНИЙ РЕЙДЕМЕЙСТЕРА}

В этом разделе мы докажем следующее утверждение.

ТеОрема 5.1. Ответъ, полученные с помощъю вычислений методом гиперкуба (6), топологически инвариантны, если выполнены свойства факторизации (10) u (11) и правила локалъных преобразований толстых графов (19)-(23), т.е. все правила из раздела 4, кроме правила флипа (24).

Чтобы доказать это, достаточно доказать, что ответы инвариантны по отношению ко всем движениям Рейдемейстера (как обычным, так и виртуальным). В следующих пунктах мы проанализируем инвариантность (6) при каждом из движений. Этот анализ требует выбора типов пересечений (черных или белых) и направлений нитей. Для экономии места мы рассмотрим только существенно разные случаи.

5.1. Первое движение Рейдемейстера. Первое движение Рейдемейстера (мы выбираем вершину черного цвета, анализ белой вершины точно такой же) имеет вид

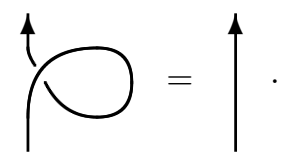

Представим, что мы производим вычисление с помощью подхода гиперкуба для некоторой диаграммы зацепления $\mathcal{L}$ с левой частью $(25)$ в форме петли. Тогда сумма (8) по всем подграфам $\gamma$ распадается на две большие суммы: ту, где ребра, связанные с пересечением в этой петле, сохранены, и ту, где ребра обрезаны (здесь мы подразумеваем суммирование по сохранению/обрезанию всех других ребер толстого графа и не рисуем их явно):

$$
\mathcal{H}_{\mathrm{lhs}}=(-q) \cdot \uparrow \cdots+\uparrow
$$


Теперь, применив правило $[N-1](19)$ и правило $[N](13)$, мы видим, что сумма равна

$$
\mathcal{H}_{\mathrm{lhs}}=((-q) \cdot[N-1]+[N]) \cdot \uparrow=q^{1-N} \uparrow
$$

На первый взгляд, сумма (8) в правой части содержит всего одну группу слагаемых с неправильным множителем

$$
\mathcal{H}_{\text {rhs }}=\uparrow
$$

Однако также стоит помнить, что общий $q$-заряд всего гиперкуба слева $\left(n_{\bullet}-n_{\circ}\right)$ на единицу больше, так как там присутствует дополнительное черное ребро, что дает дополнительный множитель $q^{N-1}$, так что на самом деле ответ устроен следующим образом:

$$
\mathcal{H}_{\text {lhs }}=\mathcal{H}_{\text {rhs }} .
$$

Если исходная вершина в (25) белая, то горизонтальное ребро в первом слагаемом в $(26)$ сплошное, в результате оно входит с коэффициентом $(-q)^{-1}$; при этом общий множитель в $(27)$ равен $(-q)^{-1} \cdot[N-1]+[N]=q^{N-1}$. В то же время полный $q$-заряд гиперкуба в левой части $\left(n_{\ldots}-n_{-}\right)$в этом случае на единицу меньше, и общий множитель равен $q^{1-N}$, таким образом, соотношение (29) снова верно.

5.2. Второе движение Рейдемейстера с параллельными нитями. Второе движение Рейдемейстера с параллельными нитями устроено как

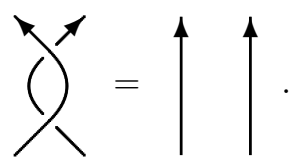

Важно, что одна из вершин планарной диаграммы черная, а другая белая, так что толстые графы имеют два цвета. Как и везде в данной работе, мы обозначаем черные вершины пунктирными линиями, а белые - сплошными. Нам необходимо изображать их по-разному, чтобы отследить правильный $q$-префактор. Но еще раз напомним, что квантовые размерности не зависят от раскраски ребер толстого графа. Таким образом, все тождества для толстых графов из раздела 4 верны вне зависимости от раскраски ребер.

На этот раз сумма по гиперкубу в левой части состоит из четырех групп слагаемых,

$$
\mathcal{H}_{\mathrm{lhs}}=1 \cdot \uparrow+(-q) \cdot \uparrow \ldots+\ldots+\hat{\uparrow}+\left(-\frac{1}{q}\right) \cdot \uparrow+1 \cdot \uparrow+
$$


тогда как сумма по гиперкубу в правой части все еще содержит только одну группу слагаемых (дополнительный множитель, связанный с изменением заряда всего толстого графа, в этот раз равен единице, так как добавлены одна черная и одна белая вершины):

$$
\mathcal{H}_{\mathrm{rhs}}=1 \cdot \uparrow \uparrow
$$

Применяя правило [2] (20) к первой группе в $\mathcal{H}_{\text {lhs }}$, замечаем, что первые три группы взаимно сокращаются и, таким образом, левая часть совпадает с правой.

5.3. Второе движение Рейдемейстера с антипараллельными нитями. Второе движение Рейдемейстера с антипараллельными нитями устроено как

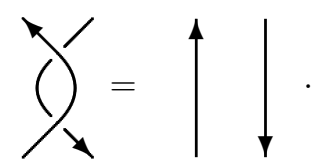

На этот раз сумма по гиперкубу в левой части вновь состоит из четырех групп слагаемых,

$$
\mathcal{H}_{\mathrm{lhs}}=1 \cdot \overbrace{+}^{1}+\left(-\frac{1}{q}\right) \cdot \overbrace{+1}
$$

а сумма по гиперкубу в правой части - из одной группы слагаемых (общий множитель, связанный с зарядом всего толстого графа, опять-таки отсутствует):

$$
\mathcal{H}_{\mathrm{rhs}}=1 \cdot \uparrow \uparrow
$$

Применяя правило $[N-2](21)$ к первой группе в $\mathcal{H}_{\mathrm{lhs}}$, правило $[N-1](19)$ ко второй и третьей группам и правило $[N](13)$ к четвертой группе и используя простое тождество для $q$-чисел

$$
[2][N-1]=[N]+[N-2],
$$

можно увидеть, что в этом случае также $\mathcal{H}_{\mathrm{lhs}}=\mathcal{H}_{\mathrm{rhs}}$.

5.4. “Параллельное” третье движение Рейдемейстера. Так как в третьем движении Рейдемейстера задействовано три нити, описывать их взаимную ориентацию как "параллельную" или "антипараллельную" не совсем точно.

Тем не менее имеются два существенно разных выбора ориентации диаграммы для третьего движения Рейдемейстера: один выбор содержит дополнительный цикл Зайферта, а другой не содержит. Проверка инвариантности различна для этих двух 
случаев, но в точности совпадает для ориентаций, которые принадлежат к одному типу. В данном пункте мы рассмотрим ориентацию, которая не содержит дополнительного цикла Зайферта (мы называем ее "параллельной” для краткости).

Один из типичных представителей такого класса ориентаций устроен как

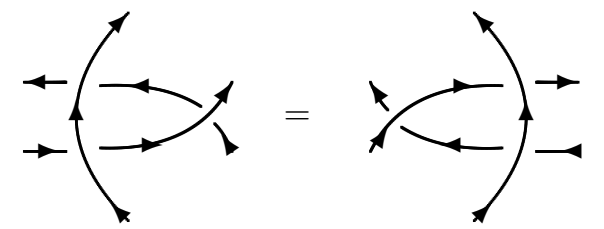

Полные заряды гиперкубов в правой и левой части совпадают. Выпишем в явном виде различные группы вкладов (опять-таки во всех изображениях мы опускаем остальную часть толстого графа, как и суммирование по обрезанию всех остальных его ребер, хотя оно, конечно, предполагается, поэтому каждое из слагаемых в суммах на самом деле является группой слагаемых; при этом важно, что они одинаковы с обеих сторон движений Рейдемейстера):

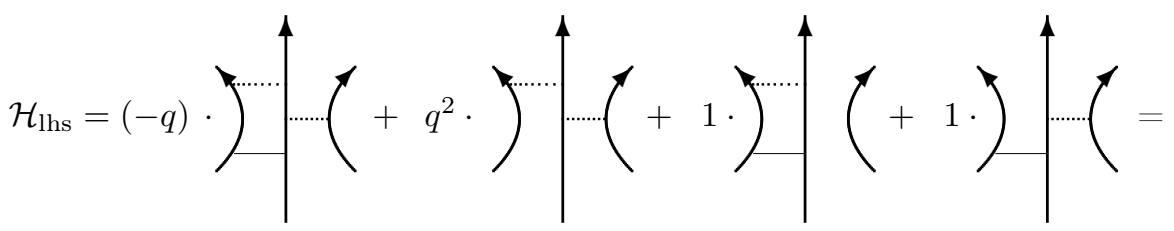

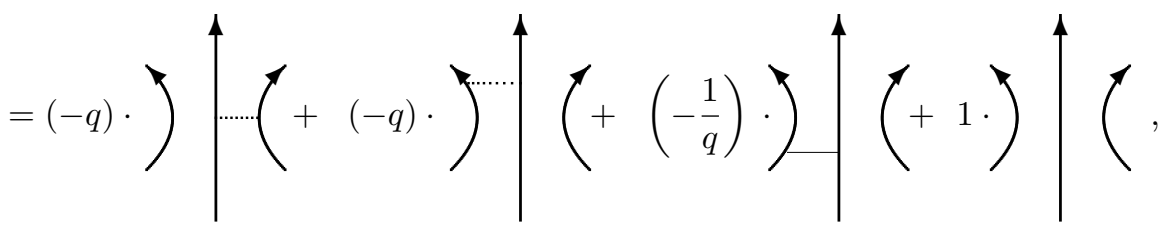

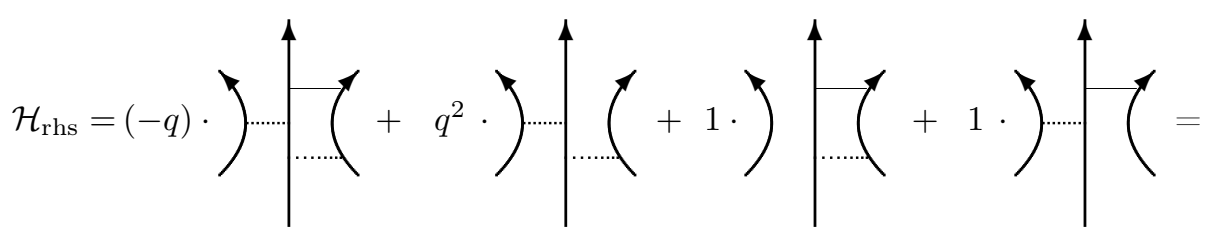

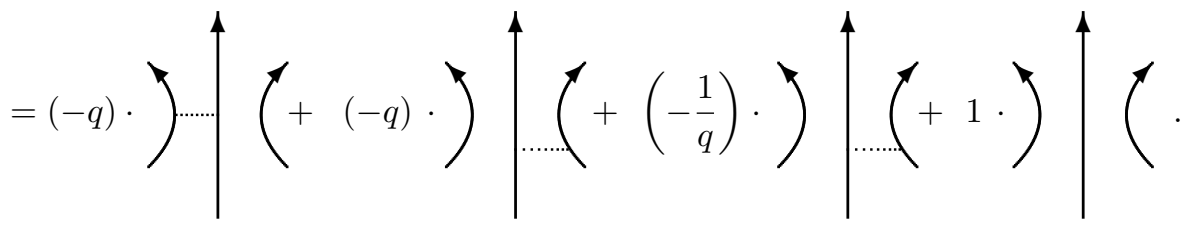

Можно заметить, что если применить правило [2] (20) к третьей группе слагаемых $\left(\right.$ в $\mathcal{H}_{\mathrm{lhs}}$ и $\left.\mathcal{H}_{\mathrm{rhs}}\right)$, то они сокращаются с шестой и седьмой группами. Более того, 
вторая, четвертая и восьмая группы в $\mathcal{H}_{\mathrm{lhs}}$ и $\mathcal{H}_{\mathrm{rhs}}$ совпадают. Может показаться, что четвертые группы отличаются (так как в левой части правое ребро пунктирное, а в левой сплошное), но напомним, что раскраска ребер на самом деле не влияет на $q$-размерности. Наконец, оставшиеся группы совпадают в силу правила 1 (23).

\section{5. “Антипараллельное” третье движение Рейдемейстера. Типичный} представитель "антипараллельного" третьего движение Рейдемейстера (т. е. того, в котором возникает дополнительный цикл Зайферта) устроен как

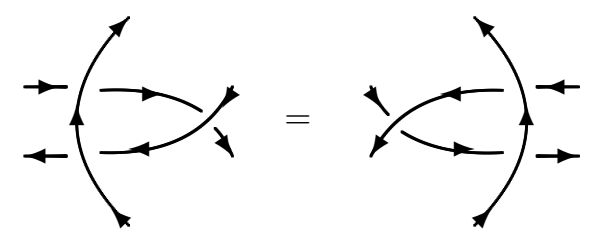

Полные заряды левой и правой частей вновь совпадают. Если явно выписать все группы слагаемых, то получается следующее:

$$
\text { (-q) }
$$

Относительно нетривиальной частью проверки является то, что после применения правила $[N-2](21)$ ко второй, третьей и четвертой группам, правила $[N-1](19)$ 
к пятой, шестой и седьмой группам и правила $[N](13)$ к последней группе, получа-

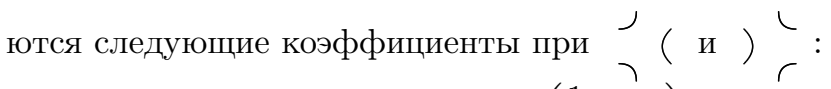

$$
\left(q^{2}+2\right)[N-2]-\left(\frac{1}{q}+2 q\right)[N-1]+[N]=q[N-3] .
$$

Если применить правило $[N-3]$, то левая и правая части совпадут и в этом случае.

5.6. Все виртуальные движения Рейдемейстера. Инвариантность по отношению ко всем четырем виртуальным движениям Рейдемейстера
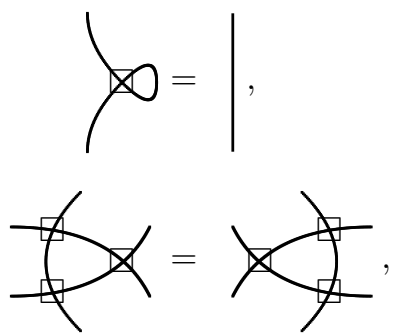
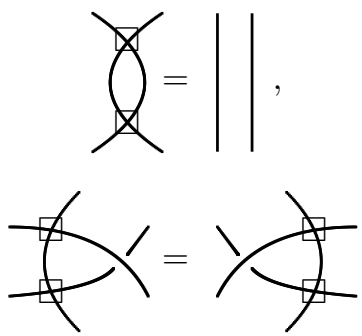

на самом деле является тривиальным фактом. Заметим, что (вне зависимости от выбора ориентаций) “основной” двуцветный толстый граф $\Gamma^{\mathcal{L}_{c}}$ вообще не изменяется при применении этих преобразований к диаграмме зацепления $\mathcal{L}$.

В частности, для "смешанного" движения (включающего обычное и виртуальные пересечения), несмотря на то что набор циклов Зайферта может быть двух типов в зависимости от ориентации нитей, а именно

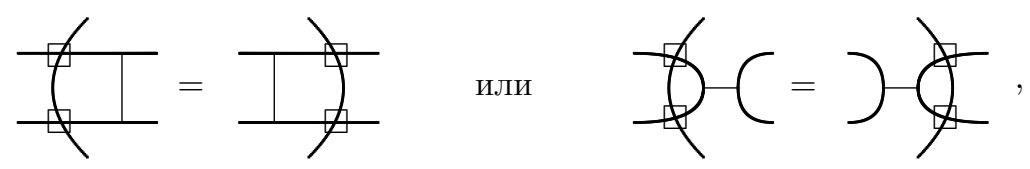

в обоих случаях наборы циклов слева и справа совпадают.

ЗАмЕчАниЕ 2. В отличие от этих выполняющихся виртуальных движений Рейдемейстера то, которое не выполняется (явным образом запрещенное в исходной конструкции Кауффмана),

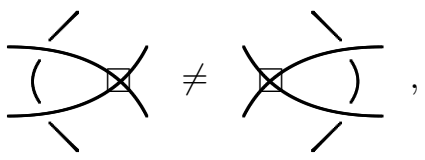

изменяет структуру циклов Зайферта. Например, для такого конкретного выбора направлений нитей правая и левая части устроены соответственно как
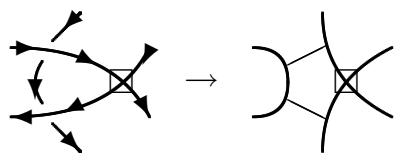

И

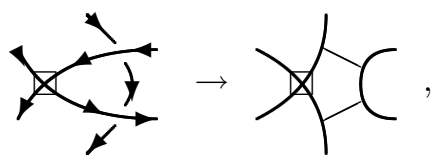

т. е. абсолютно различны. 
Возвращаясь к выполняющимся движениям Рейдемейстера, заметим, что ни правая, ни левая части равенства (8) не изменяются при этих движениях, что завершает доказательство теоремы.

\section{6. КОМПЬЮТЕРНАЯ ПРОГРАММА}

Глядя на локальные преобразования толстых графов из раздела 4, невозможно не задуматься о том, позволяют ли они вообще вычислять размерности, так как некоторые из них, (22)-(24), не упрощают толстые графы. В данном разделе мы объясняем, как работает наша программа ${ }^{2)}$; более точно, как работают версии под git-тэгами master-dmdims и flipless-dmdims.

6.1. Версия "flipless". Для начала опишем версию "flipless" (т. е. ту, которая не использует правило флипа (24)), так как она проще.

После того как диаграмме зацепления $\mathcal{L}$ сопоставлен толстый граф $\Gamma^{\mathcal{L}_{c}}$, программа проверяет все способы обрезать ребра этого графа, добавив вклады от всех подграфов $\gamma$. Это относительно тривиально. Нетривиальным является то, как программа вычисляет размерности $D_{\gamma}$. Это можно описать следующим образом.

- В первую очередь, программа проверяет, можно ли применить упрощающие правила $[N-1](19),[2](20)$ или $[N-2](21)$ к подграфу $\gamma$, т. е. похожа ли часть подграфа $\gamma$ на левую часть соответствующих выражений. Если место, где можно применить упрощающие правила, находится, то программа незамедлительно применяет его.

- Если первый шаг не дает результатов, то программа ищет все места, где в принципе можно было бы применить правило 1 (23). После этого она осуществляет полный поиск по всем способам применения правила 1 в этих местах (включая новые места, появляющиеся после применения правила 1). Для каждого такого способа она проверяет, удовлетворяет ли граф требованиям первого шага (т. е. можно ли его упростить). Если какая-нибудь из просмотренных комбинаций правила 1 приводит к графу, который можно упростить, то она применяется. Как и можно было бы ожидать, эта процедура весьма вычислительно затратна.

- Если второй шаг не дает результатов (т.е. ни один из способов применить правило 1 не приводит к графам, которые можно упростить), программа выдает ошибку, говорящую о невозможности вычисления полинома ХОМФЛИ для данной диаграммы зацепления.

Несмотря на то что нет никаких гарантий, что такой наивный алгоритм всегда будет работать, он, однако, позволяет вычислить полиномы ХОМФЛИ из представления кос для всех полиномов из таблицы Рольфсена (хотя на это и уходит в два раза больше времени, чем у версии "master"). Неясно, получились ли такие результаты по той причине, что мы ограничили рассмотрение узлами с не более чем 10 пересечениями и/или потому, что рассматривали только представление кос. Конечно, возможно (хотя и более сложно программно) извлечь другие представления узлов из таблицы Рольфсена из пакета KnotTheory программы Mathematica, и это является одним из направлений будущих исследований. Также правило $[N-3](22)$ не используется в программе. Существуют примеры виртуальных узлов, которые не удается вычислить без этого правила, но вопрос о необходимости этого правила для вычисления невиртуальных узлов пока открыт.

\footnotetext{
2) CM. https://github.com/mabragor/cl-vknots и http://knotebook.org.
} 
6.2. Версия "master". Версия "master" близка по духу к версии "flipless" (так как в ней тоже применяется полный поиск), но она использует ряд преимуществ правила флипа.

- В первую очередь проверяется, не является ли граф “легко упрощаемым”. Граф легко упрощаем, если к нему можно применить правило $[N-1]$, правило [2] или комбинацию правила флипа с правилом $[N-1]$ (сокращение рёбра-петли).

- На втором шаге получившийся граф, который не является легко упрощаемым, преобразуется в хордовую диаграмму с помощью правила флипа (24) - в форму, в которой у графа только одна вершина. Эта форма неединственна для графа, но даже такая простая оптимизация позволяет значительно уменьшить количество различных графов, которые необходимо вычислить.

- На третьем шаге программа проверяет, является ли получившаяся хордовая диаграмма упрощаемой, по аналогии с версией "flipless"; это означает, что к графу можно применить правила $[N-1],[2]$ и $[N-2]$ и их композиции с правилом флипа.

- Если третий шаг заканчивается неудачей, то применяется действительно широкий полный поиск. А именно, сначала генерируются все хордовые диаграммы данного графа. Далее для каждой из них проверяется, существуют ли комбинации правил 1, приводящие к "упрощаемому" графу.

- Если все предыдущие шаги закончились неудачей, программа выдает ошибку.

Таким образом, в версии "master" даже те графы, которые можно вычислить, используя только графы невиртуальных узлов, как правило, вычисляются с использованием графов виртуальных узлов.

Особый интерес вызывает наблюдение за тем, как различные правила из раздела 4 соединяются вместе для вычисления квантовых размерностей, особенно когда имеется несколько разных способов применить эти правила к одному и тому же графу. Иногда ответы кажутся разными на первый взгляд, но они всегда совпадают благодаря наличию соотношений между различными $q$-числами. Это наводит на мысль о существовании скрытой структуры, благодаря которой ответы совпадают. В следующем разделе мы опишем наши попытки понять эту скрытую структуру. C некоторыми оговорками их можно считать попыткой обобщить набор движений Рейдемейстера на полный набор (не зависящих от узлов) квантовых тождеств Уорда, которые могут привести к полной топологической рекурсии, схожей с рекурсией $\mathrm{AMM} / Э О$, даже если нам неизвестна в явном виде стоящая за этим матричная модель при $q \neq 1$, что точно выражает дух подхода АММ/ЭО [13]. Связь этих тождеств с конкретной явной, зависящей от узла матричной моделью, как в работе [29], еще предстоит найти.

\section{7. В НАПРАВЛЕНИИ НАСТОЯЩИХ РЕКУРСИВНЫХ СООТНОШЕНИЙ}

Из анализа, представленного в предыдущем разделе, ясно, что наличие соотношений, которые не уменьшают сложность графа, делает нашу процедуру неоптимальной. В данном разделе мы пытаемся это исправить, факторизуя изучаемые объекты по этим неупрощающим соотношениям, чтобы получить меньшее пространство модулей всех существенно различных $q$-размерностей, для которых соотношения из раздела 4 становятся настоящей рекурсией. 
7.1. Факторизация по ковариантности флипов. Ковариантность по флипам (24)

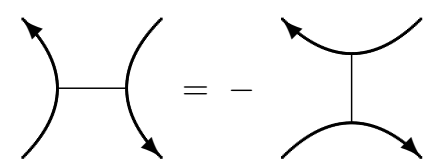

позволяет фактически сжать все ребра толстого графа в точки. После такого сокращения граф становится направленным с вершинами типа $(2,2)$ (т. е. в каждую вершину входят ровно два ребра и выходят ровно два ребра). Однако расплатой за это является потеря информации об общем знаке соответствующей $q$-размерности (напомним, что она легко может быть отрицательной для виртуальных узлов, а флипы легко переводят обычные узлы в виртуальные и обратно).

Обратное преобразование (из (2,2)-графа в толстый граф) определено неоднозначно, тем не менее мы можем канонически задать $q$-размерности для некоторых (2,2)-графов Г с помощью соотношения

$$
D_{\Gamma}=(-1)^{\#(\text { связанных компонент) }}(-1)^{\#(\text { циклов Зайферта) }} D_{\Gamma_{\mathrm{f}}},
$$

где $\Gamma_{\mathrm{f}}-$ это любое преобразование графа Г в толстый граф.

ПримеР. Для толстого графа узла $4_{1}$
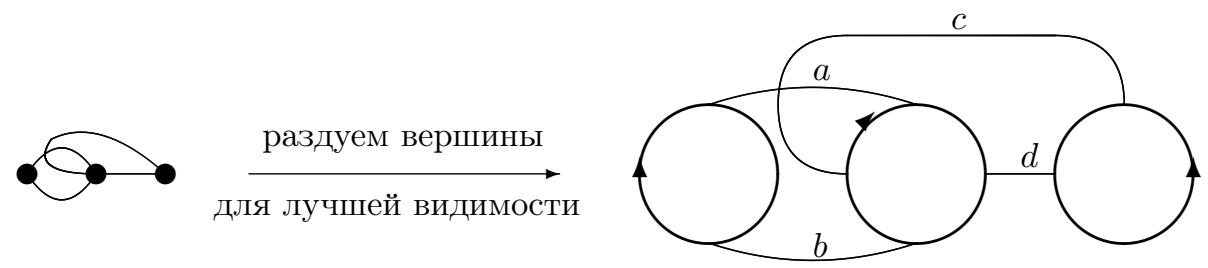

редукция до $(2,2)$-графа устроена как

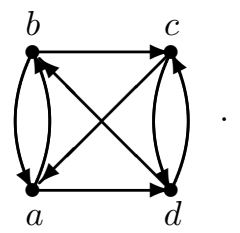

Она, конечно, согласуется как $(2,2)$-граф с исходной диаграммой зацепления узла $4_{1}$, однако нужно сделать следующие важные замечания:

- чтобы построить $(2,2)$-граф из толстого графа, не обязательно строить сначала диаграмму зацепления;

- отсутствует информация о том, как этот $(2,2)$-граф следует в точности расположить на плоскости, чтобы его можно было интерпретировать как диаграмму зацепления какого-то узла/зацепления;

- в частности, отсутствует циклический порядок входящих/исходящих ребер.

Так как число циклов Зайферта в детском рисунке для $4_{1}$ равно трем, а число связных компонент равно единице, каноническая размерность $(2,2)$-графа равна

$$
D_{\Gamma_{4_{1}}}=[N][N-1]^{2}+[N-2][2][N][N-1] .
$$


7.2. Разъяснение: рекурсивные соотношения. Теперь мы понимаем, что размерности по сути зависят только от структуры $(2,2)$-графа, и заманчиво работать только с $(2,2)$-графами, полностью забыв о толстых графах, однако на данный момент это невозможно. Это связано с тем, что толстые графы, возникающие в рекурсивных соотношениях, вообще говоря, могут иметь несколько связных компонент, отличных друг от друга. Таким образом, при проекции соотношений на $(2,2)$-графы появляются дополнительные знаки, которые зависят от конкретного рассматриваемого графа.

Пример. Рассмотрим удаление разнонаправленных ребер (комбинацию применения двух правил флипа и правила $[N-2])$ :

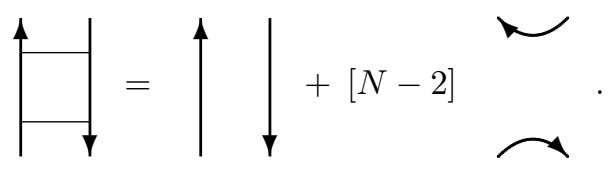

При применении к хордовой диаграмме с двумя непересекающимися нитями

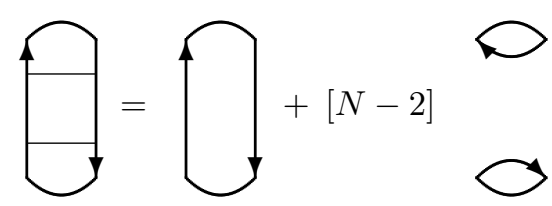

эта процедура приводит к соотношению на уровне $(2,2)$-графов без каких-либо новых знаков (так как изменение числа циклов Зайферта компенсируется изменением числа связных компонент):

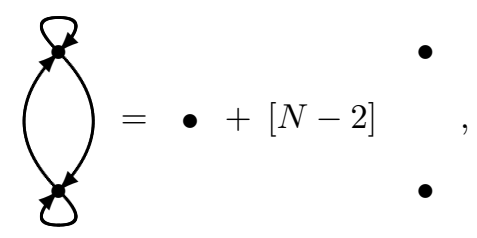

$$
[N](-1)^{2}[N-1]^{2}=[N]+[N-2][N]^{2} .
$$

Однако если мы добавим еще одно ребро так, что все толстые графы в рекурсии останутся соединенными,

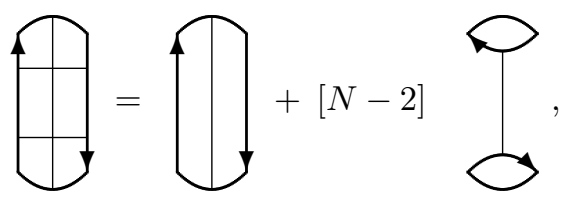


то дополнительный знак возникает на уровне соотношений между $(2,2)$-графами:

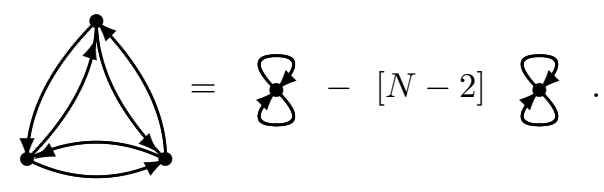

Таким образом, несмотря на то что с целью каталогизации можно использовать $(2,2)$-графы, при вычислении размерностей нам всегда необходимо возвращаться к структурам толстых графов.

7.3. Редукция соотношений к $(2,2)$-графам: свобода знаков. Возможно, однако, пойти и немного дальше в рассмотрении $(2,2)$-графов и сформулировать рекурсии в терминах этих графов, но с неизвестными дополнительными переменными $\epsilon_{i}$, принимающими значения \pm 1 . Значения этих переменных можно позже установить, налагая требование правильного поведения в пределе $q \rightarrow 1$. Но, вообще говоря, неясно, всегда ли классический предел позволяет исправить такую неопределенность, вне зависимости от сложности рассматриваемого графа. Это, безусловно, является одним из направлений будущих исследований. Приведем здесь данные проекции правил с "неизвестными знаками" $\epsilon_{i}$.

Правило $[N-1](19)$ переходит в

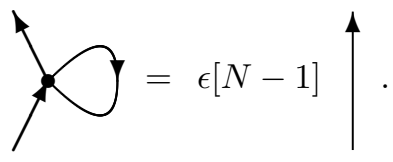

Правило [2] (20) переходит в

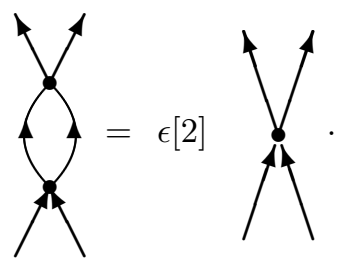

Правило $[N-2](21)$ переходит в

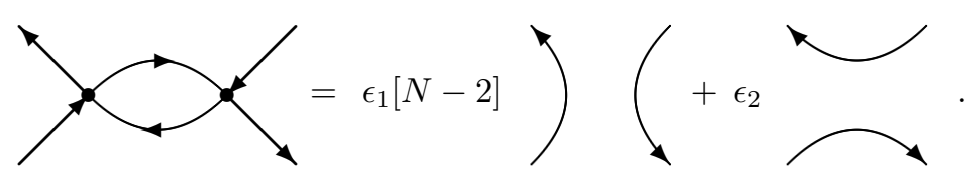


Правило 1 (23) переходит в

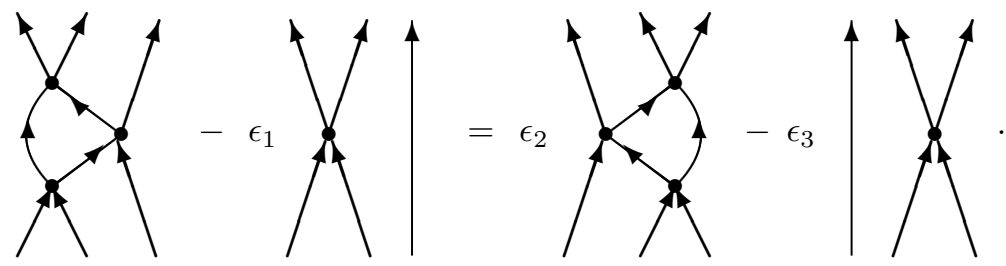

Правило $[N-3](22)$ переходит в
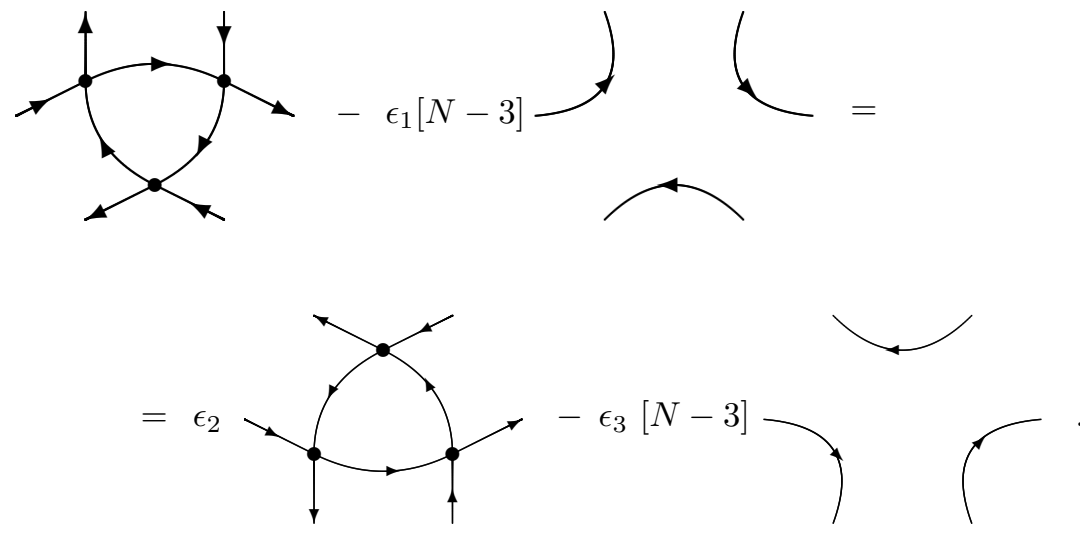

Естественно, на практике нет необходимости отслеживать все различные $\epsilon_{i}$, появляющиеся на каждом шаге разложения. Достаточно только добавлять новую неизвестную фазу $\epsilon_{i}$ каждый раз, когда рекурсия приводит к пустому толстому графу.

7.4. Факторизация по правилу 1: вершины высокой валентности. Даже после редукции к $(2,2)$-графам мы еще не достигли своей цели: применение правила 1 и правила $[N-3]$ не упрощает графы. Мы можем попробовать обойти эту трудность для правила 1, объявив левую и правую стороны соотношения (23) равными вершине большей валентности - $(3,3)$-вершине (здесь и далее мы опускаем знаковые множители $\epsilon$, но они присутствуют в каждом преобразовании графов):
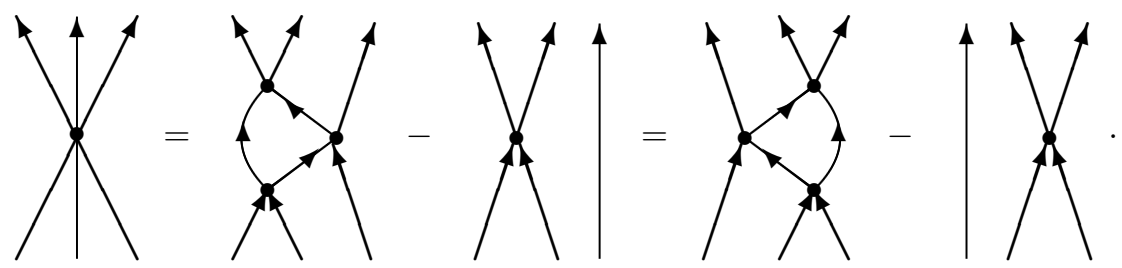
Отсюда после небольших усилий можно понять, что все проекторы на абсолютно антисимметрические представления на этом рисунке становятся вершинами большей валентности (см. обозначения антисимметрических проекторов и другие полезные формулы при $q=1$ в п. 6.2 работы [30]):
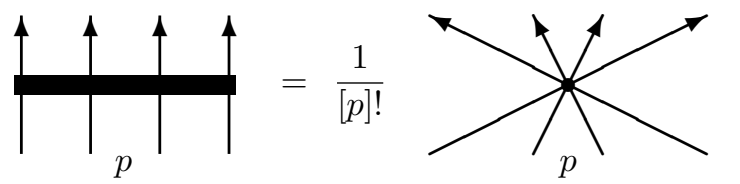

Соотношение между $(n-1, n-1)$-вершиной и $(n, n)$-вершиной (проекция соотношения между проекторами на полностью антисимметрические представления) выглядит естественно (для случая $q=1$ оно опять-таки приведено в статье [30]), но для получения формулы, которую можно обобщить на случай $q \neq 1$, необходимо заменить перестановку разностью между единицей и проектором на представление [1,1]):

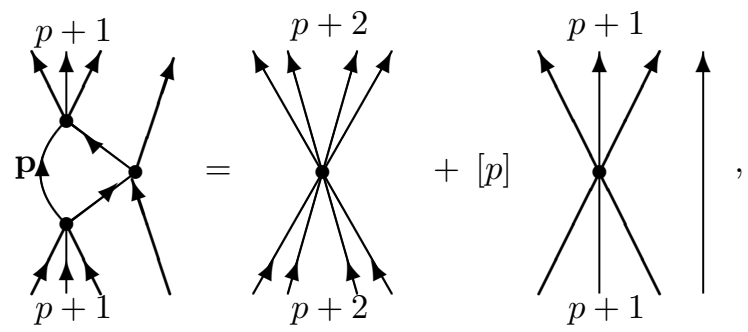

если ввести нормированные мультиребра (мы обозначаем множественность ребра жирными переменными, чтобы избежать путаницы в обозначениях). Иногда мы также называем множественности импульсами, так как они сохраняются в каждой вершине:

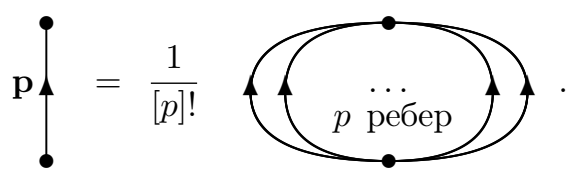

Удобно считать ребро с нулевым импульсом отсутствующим, а ребра с отрицательными импульсами - нулевыми; размерности графов с такими ребрами автоматически равны нулю:

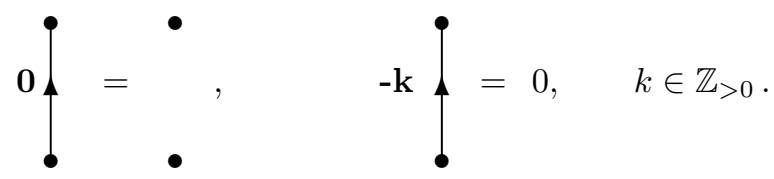


7.5. Свойство абсорбции и кластеризация ребер. Свойство абсорбции высших антисимметрических проекторов (см. работу [30], формула (6.16))

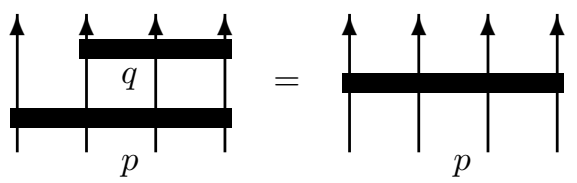

также проецируется на направленный граф. Для него оно принимает следующий вид: любая группа сонаправленных стрелок может быть "отделена" от любой вершины с помощью добавления промежуточного ребра (импульс этого ребра таков, чтобы импульсы сохранялись во всех вершинах):

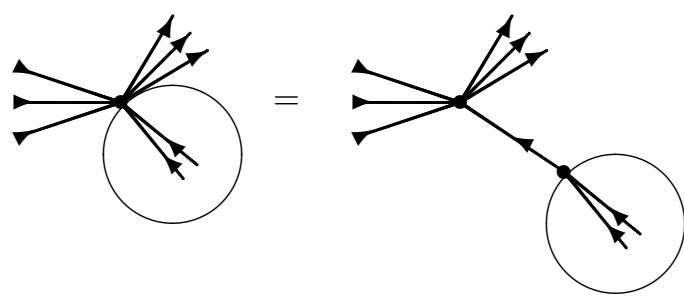

Отсюда вытекает важное следствие: для любого рекурсивного соотношения, которое мы сформулируем, достаточно двух (одного входящего и одного исходящего) внешних ребер в каждой вершине. Например, соотношение (42) можно переписать как следующее правило сокращения беспетлевого треугольника:

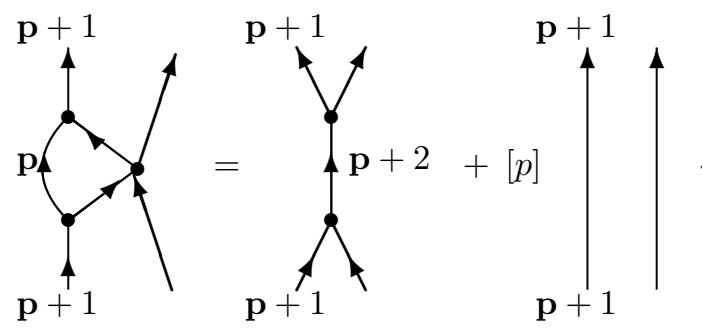

При этом соотношение в форме (42) с произвольным числом входящих и исходящих ребер автоматически следует из него. Поэтому в дальнейшем мы будем описывать все соотношения в такой краткой форме.

Направленные графы с мультивалентными вершинами и импульсами на ребрах выглядят красиво. Что более важно, правило 1 в этом случае представляет собой редукцию к (3,3)-валентным вершинам; тем самым мы сделали еще один шаг в направлении настоящих рекурсивных соотношений. Однако за это тоже приходится платить: теперь мы расширили пространство объектов, с которыми работаем, и пока нам не хватает соотношений для вычислений в этом пространстве. Мы должны как-то их вывести (или угадать дополнительные) из базовых соотношений для (2,2)-графов из п. 7.3.

Следующий раздел описывает наш прогресс в поисках таких эффективных соотношений, но это пока незаконченный набор. 


\section{6. Рекурсивные соотношения для направленного импульсного графа.} В этом пункте мы приводим соотношения для направленных мультивалентных графов с импульсами (мотивировка введения таких графов была изложена в предыдущем пункте). Для тех соотношений, которые можно получить из соотношений для $(2,2)$-графов, мы даем подсказку, как это можно сделать. Мы также указываем, для каких соотношений это сделать нельзя (или мы не знаем как).

Прежде всего, след ребра (вследствие (43) и (42)) имеет вид

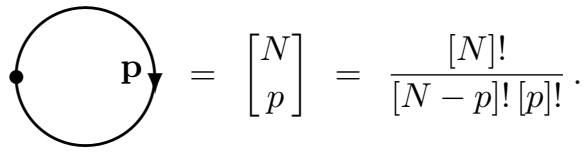

Более того, такое же уравнение приводит к формуле сокращения любого 1-цикла:

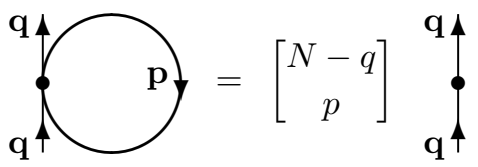

На языке направленных графов правило $1 /[N]$-разложения (11) превращается в следующее (оно не выводится откуда-либо):

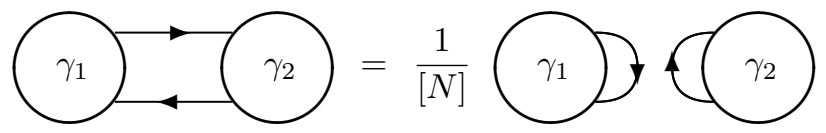

(графы $\gamma_{1}$ и $\gamma_{2}$ здесь предполагаются соединенными только посредством двух ребер, которые указаны на рисунке). Его импульсное обобщение устроено как (графы $\gamma_{1}$ и $\gamma_{2}$ опять-таки соединяются только посредством двух мультиребер)

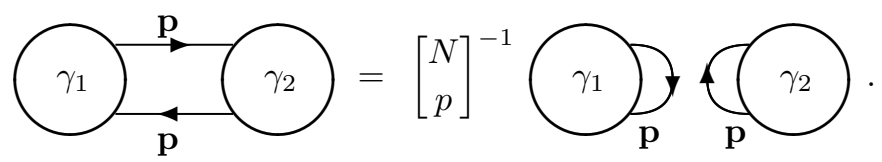

Эта формула представляет особый интерес, так как при $p=0$ она содержит свойство факторизации (10), а при $p=1$ - свойство факторизации (11) (если учесть соотношение (44)).

7.6.1. Рекурсия по дигонам. Дигон с оориентированными сторонами всегда может быть сокращен, вне зависимости от других входящих и исходящих ребер в его вершинах (просто по определению мультиребер):

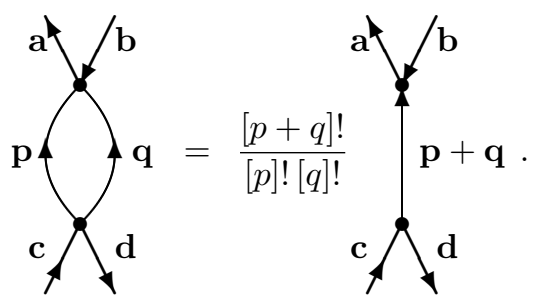


Для дигонов с разнонаправленными ребрами (таких, которые вводят в граф петли) из правила разложения (45) мы получаем

$$
\mathbf{p}+\mathbf{q}
$$

Когда в каждой вершине дигона присутствуют как входящие, так и исходящие ребра, но импульс в обеих вершинах равен единице, формула разложения является следствием правила $[N-2](21)$ :
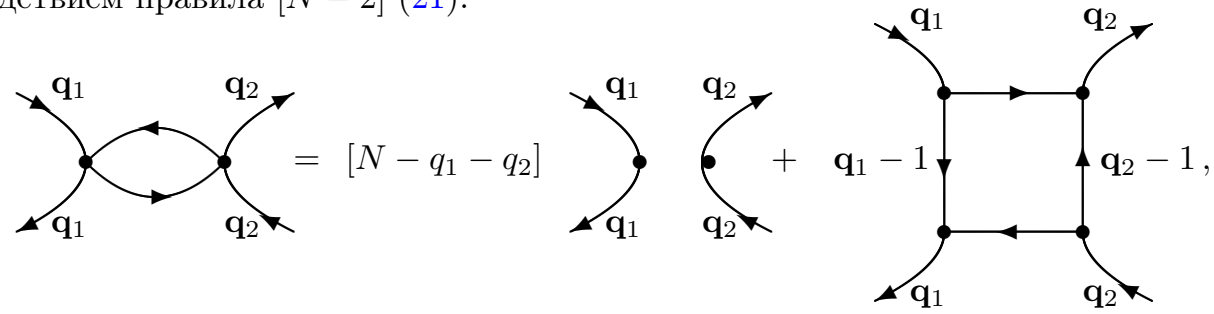

Данное соотношение для дигона с мультиребрами предположительно принимает следующую форму:

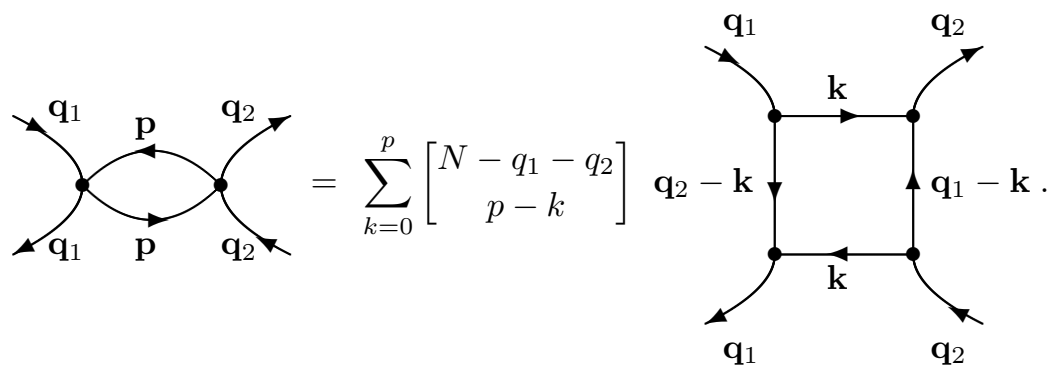

Опять-таки предположительно, при переносе импульса между вершинами дигона, оно принимает вид

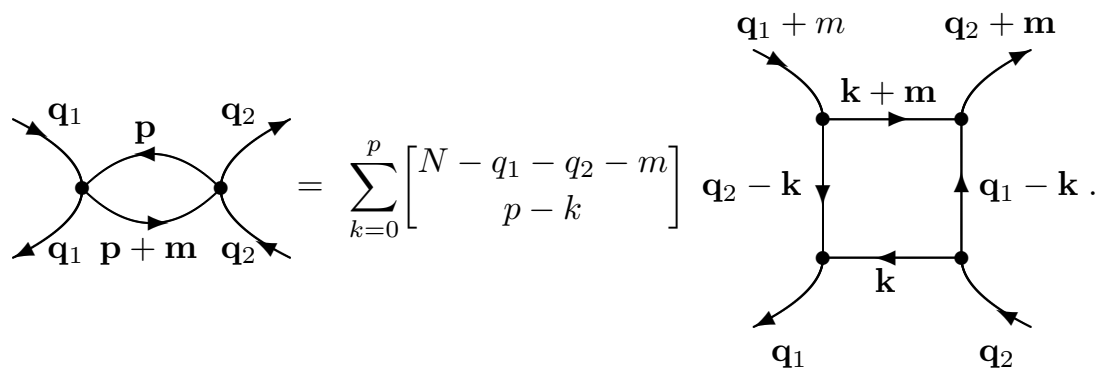

Можно задаться вопросом, в каком смысле выражения (46) и (47) являются рекурсиями, так как диаграммы в правой части выглядят более сложными. Но на самом деле у них либо строго меньшая полная сумма импульсов (при всех $k \neq 0$ ), либо они структурно проще из-за наличия ребер с нулевым импульсом (случай $k=0$ ). 
7.6.2. Тригональная (треугольная) рекурсия. С помощью повторных применений соотношения (42) между вершинами высокой валентности легко получить рекурсивные соотношения для уничтожения беспетлевого тригона:

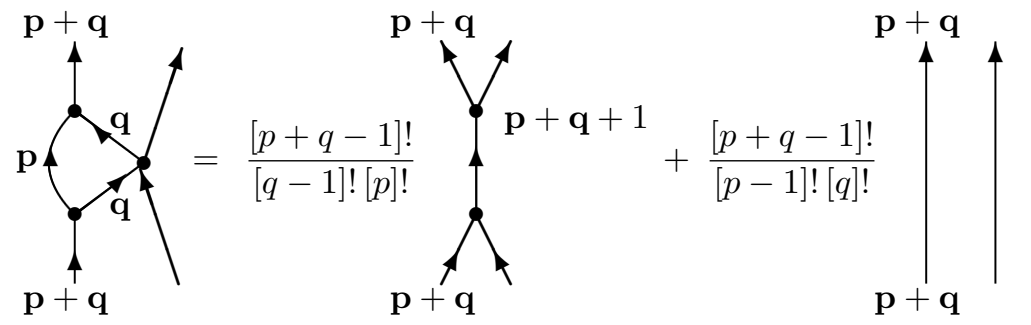

Более или менее аналогично рекурсивным формулам для дигона можно предположить, что имеется обобщение на случай, когда правые ребра обладают импульсом $m$ (такая конфигурация проходит элементарную проверку с помощью следа):

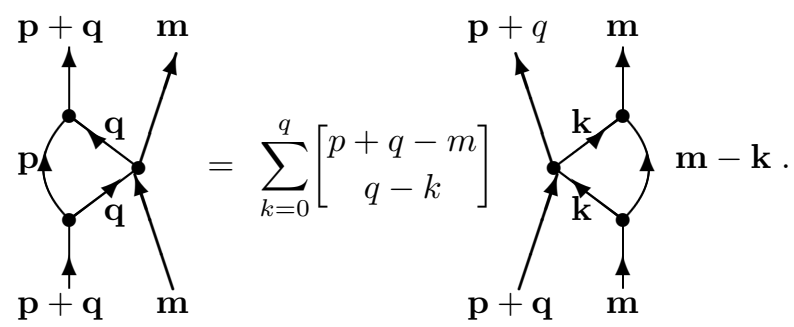

7.6.3. Общие наблюдения и замечания. Во-первых, создается ощущение, что, когда мы разбиваем петлю с помощью рекурсии, в коэффициентах возникает $N$, в противном случае не возникает. Во-вторых, общая структура устроена, по-видимому, так, что подграф (с некоторыми входящими и исходящими стрелками) может быть заменен суммой по всем возможным переносам импульса между этими внешними ребрами с некоторыми коэффициентами. Тем не менее пока неясно, как устроена общая формула для этих коэффициентов.

Эти замечания завершают описание достигнутого нами прогресса в понимании полной структуры рекурсии в случае $q \neq 1$. Работа над улучшением нашего понимания продолжается.

ПРИЛОЖЕНИЕ А Об отображении $\{$ диаграмма зацепления $\} \rightarrow\{$ толстый граф\}

А.1. Преобразование не инъективно: "развязывание" Кишино. Здесь мы продемонстрируем, что две различные диаграммы зацепления могут соответствовать одному и тому же толстому графу. Для того чтобы сделать пример более впечатляющим, мы рассмотрим известный пример узла Кишино. Его диаграмма зацепления и соответствующий двухцветный граф устроены как

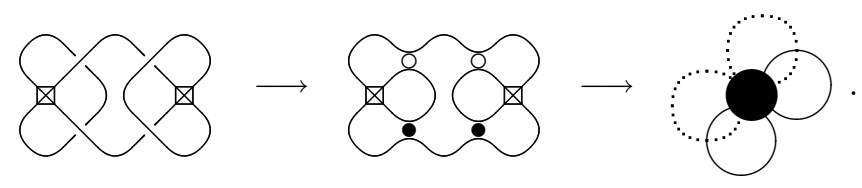


Теперь заметим, что если мы рассмотрим другую композицию двух виртуальных трилистников из узла Кишино, то подкомпоненты графы останутся такими же (включая и расцветку графа):

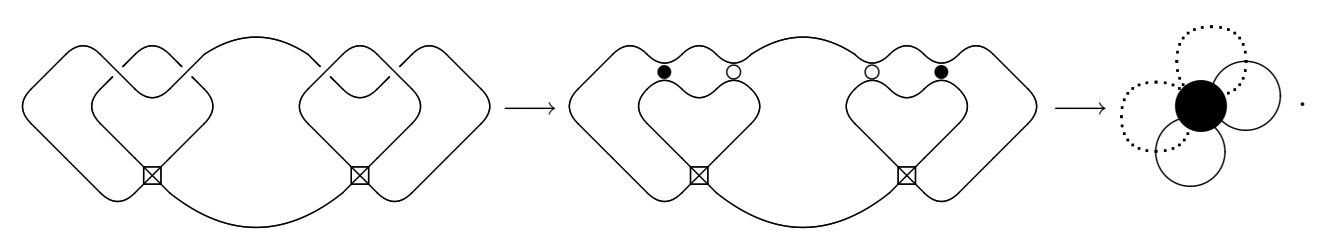

Тем не менее два вторых движения Редемейстера с последующими двумя первыми виртуальными движениями Редемейстера распутывают вторую комбинацию, тогда как известно, что узел Кишино нетривиален.

А.2. Преобразование сюръективно: алгоритм спиралей и арок. Здесь мы опишем идею алгоритма, который позволяет построить какую-нибудь исходную диаграмму зацепления по произвольному толстому графу. Эта диаграмма зацепления может не являться (обычно так и бывает) простейшей диаграммой с таким толстым графом. Мы также не формулируем алгоритм строго, приводя вместо этого пару примеров того, как он должен работать. Мы верим, что выработка всех деталей вплоть до уровня написания явных формул для графа диаграммы зацепления определенно возможна (хотя и требует больших трудозатрат). Поэтому написание компьютерной программы, которая по заданному толстому графу выдает команду для рисования диаграммы зацепления в LaTeX, не вызывает теоретических затруднений.

Идея алгоритма состоит в следующем.

- Каждая вершина толстого графа превращается в спираль, расположенную в кольце $2 i<r<2 i+1$ (для некоторой произвольной нумерации вершин), с отдельными "выступами" к центру круга.

- Каждое ребро толстого графа превращается в арку в единичном круге, соединяющую точки

$$
r=1, \quad \phi=\pi \frac{2 j}{\#(\text { ребер })} \quad \text { и } \quad r=1, \quad \phi=\pi \frac{2 j+1}{\#(\text { ребер })}
$$

(опять-таки для некоторой произвольной нумерации ребер).

- Наконец, каждая арка превращается в комбинацию пересечение + виртуальное пересечение (так как части циклов Зайферта около арок противоположно направлены).

Этот алгоритм, примененный к толстому графу виртуального трилистника, дает (мы опускаем квадраты у виртуальных пересечений, так как их слишком много на 
этом рисунке) следующее:
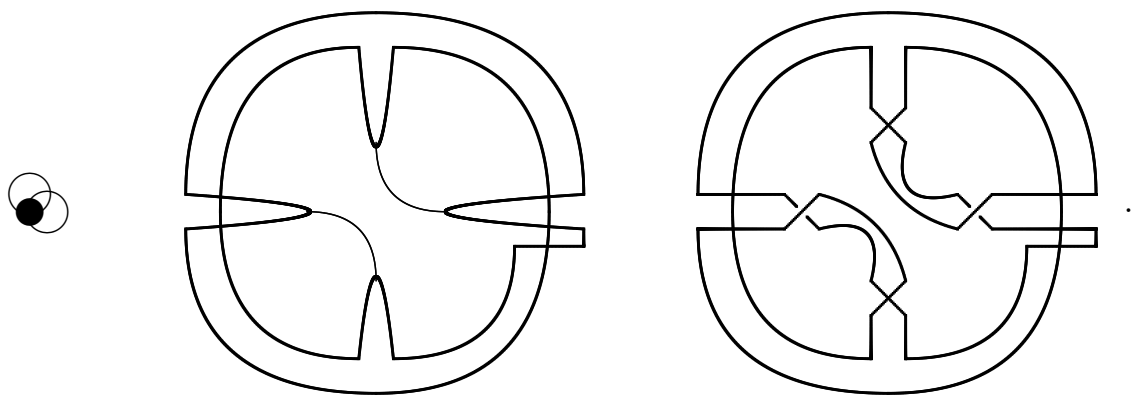

При этом алгоритм, примененный к толстому графу перекрученного неузла, дает
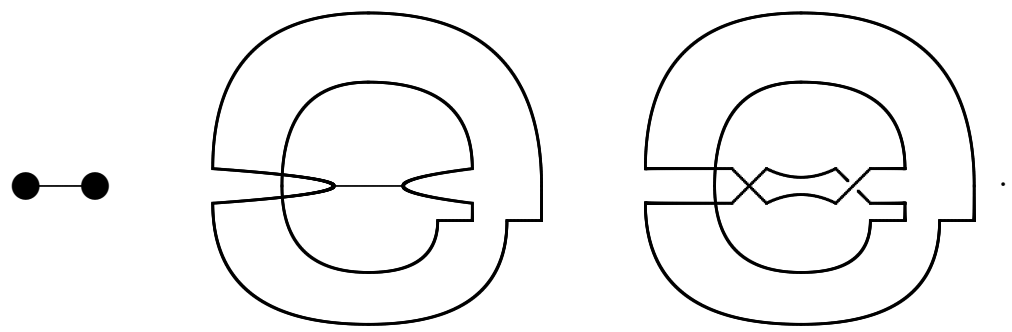

Конечно, с ростом числа ребер выступы становятся все более острыми, так как им необходимо поместиться в один сектор размера $\pi$ / \#(ребер), что делает этот метод рисования диаграмм зацеплений не очень практичным. Тем не менее он позволяет продемонстрировать существование по крайней мере одной диаграммы зацепления, которая отображается на данный толстый граф.

\section{Таблица размерностей}

ПРИЛОЖЕНИЕ В

В этом разделе приведен дополнительный набор примеров квантовых размерностей. Их можно использовать для проверки правил разложения из раздела 4, а также правил факторизации (10), (11). Все выражения можно вычислить следующим образом: для данной планарной диаграммы узла, для которой интересующий нас толстый граф является самым сложным графом, существует такая раскраска вершин, что этот узел является неузлом (так называемая развязывающая раскраска). Если квантовые размерности для всех меньших графов известны, то это позволяет найти выражения и для этой размерности. После этого с помощью таблицы Рольфсена можно подтвердить, что полиномы ХОМФЛИ узлов, соответствующих различным раскраскам планарной диаграммы, действительно верны. Далее везде, где это важно, указаны соответствующие планарные диаграммы узлов и развязывающая окраска. Все размерности ниже не нормированы (т. е. не поделены на $[N]$ ).

В.1. Выдержки из работы [23]. В работе [23] применялся формализм РТ, в рамках которого результаты можно получить только для обычных (невиртуальных) узлов и зацеплений. 
Размерности определяются проекторами $\pi_{1}, \ldots, \pi_{m-1}$ и зависят от слов из $m-1$ букв, где одинаковые буквы никогда не стоят рядом друг с другом:

$$
V_{n, \vec{a}}=[2]^{n} \operatorname{Tr}\left(\widehat{D} \pi_{a_{1}} \pi_{a_{2}} \ldots\right) .
$$

Здесь $m$ - это число нитей (т. е. число циклов Зайферта и вершин в нашем графе), a $n$ - это число пересечений диаграммы зацеплений (т. е. число ребер в графе). Оператор $\widehat{D}$ отвечает за градуировку: он получается из градуированных размерностей неприводимых представлений $[1]^{m}$, мы часто включаем его в определение следа. Мы используем заглавную букву $V$, чтобы отметить, что в данном разделе размерности не нормированы (т. е. включают в себя общий множитель $[N]$ ).

В.1.1. Случай $m=1$. В этом случае не существует невиртуальных узлов, кроме неузла с $V_{\varnothing}=[N]$.

В.1.2. Случай $m=2$. В этом случае имеется только одна буква и, таким образом, одно слово (так как буква не может повторяться):

$$
V_{n}=[2]^{n} \operatorname{Tr} \pi=[2]^{n} \frac{[N][N-1]}{[2]} .
$$

В.1.3. Случай $m=3$. В случае трех нитей имеются две буквы, но слова все еще просто классифицируются: единственному типу связных графов соответствует

$$
\begin{aligned}
V_{n, k} & =[2]^{n} \operatorname{Tr}\left(\pi_{1} \pi_{2}\right)^{k}=2^{n} \frac{[N][N-1]\left([N-2]+2^{1-2 k}[N+1]\right)}{[2][3]}= \\
& =[2]^{n-2 k}[N][N-1]\left([N-1]+[2][N-2] \sum_{i=0}^{k-2}[2]^{2 i}\right) .
\end{aligned}
$$

В.1.4. Случай $m=4$. В этом случае имеются три буквы, и классификация слов уже достаточно сложна (см. несколько примеров в формулах (6.16), (6.17) из работы [23]; отметим также опечатки в этих формулах: в $v_{n k}$ должны быть слагаемые $[2]^{2 i}$ вместо $\left.[2]^{i}\right)$. В частности, “торические" размерности устроены как

$$
\begin{aligned}
V_{n,(123)^{k}}= & {[2]^{n} \operatorname{Tr}\left(\pi_{1} \pi_{2} \pi_{3}\right)^{k}=[2]^{n-2 k-2}[N][N-1] \times } \\
& \times\left([2][N-1]^{2}+2\left(2^{k-1}-1\right)[N-1][N-2]+\xi_{k}[N-2][N-3]\right),
\end{aligned}
$$

где $\xi_{k}$ задаются следующим образом:

$$
\begin{array}{rlrl}
k & =1: & & 0, \\
k=2: & & 1, \\
k & =3: & & {[3]+4,} \\
k & =4: & & {[5]+6[3]+12,} \\
k & =5: & & {[7]+8[5]+25[3]+33,} \\
k & =6: & & {[9]+10[7]+42[5]+91[3]+89,} \\
k & =7: & & {[11]+12[9]+63[7]+185[5]+313[3]+243, \quad \ldots,}
\end{array}
$$


другими словами,

$$
\begin{gathered}
\xi_{k}=\sum_{j=0}^{k-2}[2 k-3-2 j] u_{k, j}, \\
u_{k, 0}=1, \quad u_{k, 1}=2 k-2, \quad u_{k, 2}=k(2 k-5), \quad \ldots . .
\end{gathered}
$$

\section{В.2. Простейшие графы.}

B.2.1. Торические зацепления/узлы. Толстые графы для представлений кос торических узлов очень легко нарисовать. Для узла $[n, m]$ у этого графа имеются $n$ вершин и $n m$ ребер. Характерный пример - это трилистник, торический узел $[3,2]$ :

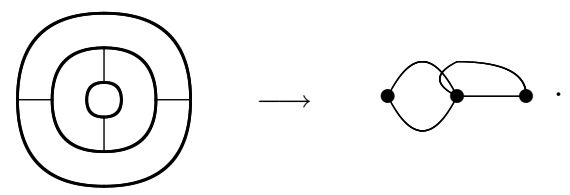

Для семейства $[2, n]$ имеем

$$
\operatorname{dim}_{[2, n]}=[2]^{n-1}[N][N-1]=[N][N-1]+\left([2]^{n}-[2]\right) \frac{[N][N-1]}{[2]} .
$$

Таким образом, можно написать, используя обозначения из п. В.1 (игнорируя множители [2]), следующее соотношение:

$$
V_{n+1}-[2] V_{n}=0, \quad\left(e^{\partial / \partial n}-[2]\right) V_{n}=0,
$$

где $e^{\partial / \partial n}-$ как обычно, оператор “сдвига", который здесь действует на индекс $n$.

Для семейства $[3, n]$ имеем

$$
\begin{aligned}
\operatorname{dim}_{[3, n]} & =[N][N-1]^{2}+(\overbrace{[2]+[2]^{3}+[2]^{5}+\cdots .}^{n-1 \text { слагаемых }})[N][N-1][N-2]= \\
& =[N][N-1]^{2}+\left([2]^{2 n}-[2]^{2}\right) \frac{[N][N-1][N-2]}{[2][3]} .
\end{aligned}
$$

Разностное уравнение в данном случае опять-таки очень простое,

$$
V_{n+1}-[2]^{2} V_{n}=[N][N-1](-[2][N]+[N-1]),
$$

и его можно перевести в знакомое по методу эволюции уравнение для оператора второго порядка:

$$
V_{n+2}-\left([2]^{2}+1\right) V_{n+1}+[2]^{2} V_{n}=0, \quad\left(e^{\partial / \partial n}-1\right)\left(e^{\partial / \partial n}-[2]^{2}\right) V_{n}=0 .
$$

Для семейства $[4, n]$ общую формулу можно предположить на основании соотношения (В.1). Соответствующее разностное уравнение имеет вид

$$
\begin{aligned}
V_{k+1}-[2]^{2} V_{k}= & {[N][N-1]\left\{\left(2^{k}-1\right)[2][N]^{2}+\right.} \\
& +\left(\left(3 \cdot 2^{k}-4\right)[2]^{2}-\left(3 \cdot 2^{k}-3\right)\right)[N][N-1]+ \\
& \left.+\left(\left(2^{k+1}-4\right)[2]^{3}-\left(3 \cdot 2^{k}-4\right)[2]\right)[N-1]^{2}\right\}
\end{aligned}
$$


и отсюда легко написать разностное уравнение третьего порядка:

$$
\begin{aligned}
V_{n+3}-\left([2]^{2}+3\right) V_{n+2}+\left(3[2]^{2}+2\right) V_{n+1}-2[2]^{2} V_{n} & =0, \\
\left(e^{\partial / \partial n}-1\right)\left(e^{\partial / \partial n}-2\right)\left(e^{\partial / \partial n}-[2]^{3}\right) V_{n} & =0 .
\end{aligned}
$$

ЗАмЕчАниЕ 3. Уравнения (В.2) представляют собой простое следствие из правил из раздела 4. Соотношения (В.3), однако, не так просто вывести из них (по крайней мере нам это не удалось). Но если рассмотреть антисимметрические проекторы высоких порядков (вершины высокой валентности), то эту формулу можно получить с помощью не таких уж длинных расчетов, надо только выразить проекторы на представление $[1,1,1,1]$ через торические диаграммы и использовать после этого правило абсорбции.

Серия $[m, 2]$ представляется графом $(m, 2 m-2, m-1, m-1)$,

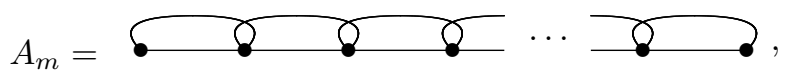

который можно использовать для проверки правила $[N-2](21)$ :

$$
A_{m+1}=[N-2] \cdot A_{m}+[N-1]^{2} \cdot A_{m-1},
$$

T. e.

$$
\begin{aligned}
A_{1}= & {[N], } \\
A_{2}= & {[2][N][N-1], } \\
A_{3}= & {[N][N-1]([N-1]+[2][N-2]), } \\
A_{4}= & {[N][N-1]\left([2][N-1]^{2}+[N-1][N-2]+[2][N-2]^{2}\right), } \\
A_{5}= & {[N][N-1]\left([N-1]^{3}+2 \cdot[2][N-1]^{2}[N-2]+\right.} \\
& \left.+[N-1][N-2]^{2}+[2][N-2]^{3}\right), \\
& \ldots \ldots \ldots \ldots \ldots \ldots \ldots \ldots \ldots \\
A= & {[N][N-1] \sum_{j=0}^{m}[N]^{m-j}[N-1]^{j} \times \ldots . } \\
& \\
& \times\left\{\begin{array}{c}
(-1)^{m-1} \sum_{i=0}^{k}\left(\begin{array}{c}
k+i \\
2 i
\end{array}\right)\left(\begin{array}{c}
m-k+i \\
k+i-1
\end{array}\right)[2]^{2 i}, \\
(-1)^{m} \sum_{i=0}^{k}\left(\begin{array}{c}
k+i+1 \\
2 i+1
\end{array}\right)\left(\begin{array}{c}
m-k+i \\
k+i
\end{array}\right)[2]^{2 i+1}, \quad j=2 k .
\end{array}\right.
\end{aligned}
$$

Для семейства $[m, 3]$, т. е. графов $(m, 3 m-3,2 m-2, m-1)$ имеем

$[1,3]: \quad[N]$,

$[2,3]: \quad[2]^{2}[N][N-1]$,

$[3,3]: \quad[N][N-1]\left([N-1]+\left([2]+[2]^{3}\right)[N-2]\right)$,

$[4,3]: \quad[2][N][N-1]\left\{[N][N-1]+[N-2]\left(4[N-1]+3[2][N-2]+[2]^{2}[N-3]\right)\right\}$, 
$[5,3]: \quad[N][N-1]\left\{[N]^{2}\left([N-1]-2[2][N-2]-4[2]^{2}[N-3]-3[2]^{3}[N-2]+\right.\right.$

$$
\begin{aligned}
& \left.+2[2]^{5}[N-4]+[2]^{6}[N-5]\right)+[N-1]+4[2][N-2]+[2]^{2}[N-3]+ \\
& \left.+11[2]^{3}[N-2]+4[2]^{4}[N-3]+[2]^{5}[N]+[2]^{6}[N-3]+[2]^{7}[N]+[2]^{9}[N]\right\}
\end{aligned}
$$

При $q=1$ величина

$$
B_{m+1}=(N-4) B_{m}+(N-1) B_{m-1}^{\prime},
$$

где $B^{\prime}$ содержит дополнительное двойное ребро, которое присоединено с перекруткой к вершине $B_{m-1}^{\prime}$, может быть вычислена с помощью (21):

$$
B_{m-1}^{\prime}=\underset{\cdots}{B_{m-1}} .
$$

При $q \neq 1$ компьютерный эксперимент показывает (проверено вплоть до $m=25$ ), что

$$
A_{n}^{(3)}=[2][N-2] A_{n-1}^{(3)}+(2+[2][N-1][N-2]) A_{n-2}^{(3)}+[N-1][N-2]^{2} A_{n-3}^{(3)}-[N-1]^{4} A_{n-4}^{(3)} \text {. }
$$

Однако нам пока не удалось вывести эту рекурсию как из правил из раздела 4, так и из вершин большей валентности, но, возможно, стоит приложить больше усилий в этом направлении.

В.2.2. Твистованные узлы. Зайфертово разрешение для нечетных и четных твистованных узлов устроено как

нечетные узлы:

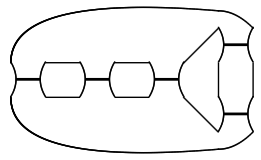

четные узлы:

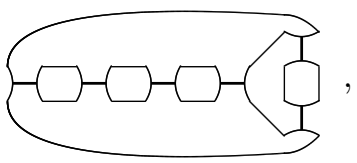

так что соответствующие толстые графы могут быть представлены в форме таких ожерелий:
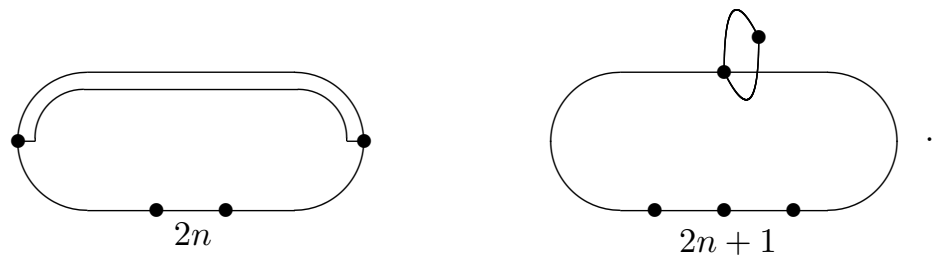
Нечетный детский рисунок очень прост: пользуясь формулами (9.3) из работы [23], можно найти простую связь с формулой для графа-ожерелья:

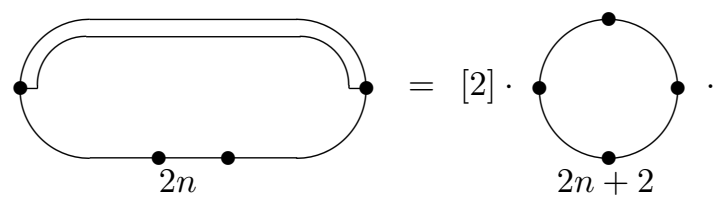

Граф для нечетных твистованных узлов несколько более сложен, его можно связать с другими графами двумя способами:

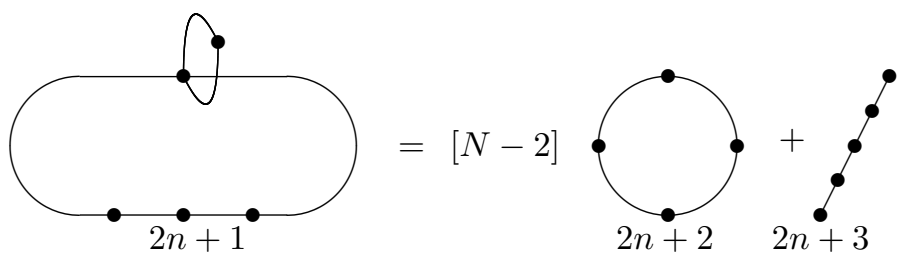

или

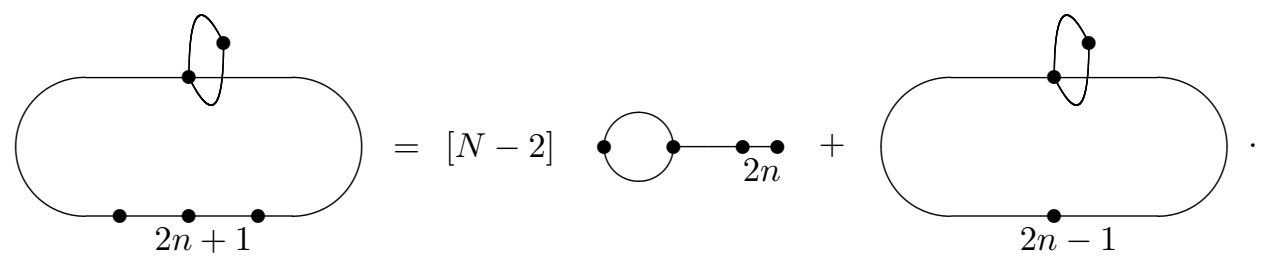

В.2.3. Претцелевы узлы/зацепления. Все параллельные узлы: циклы с четным числом пересечений с ребрами произвольной множественности суть
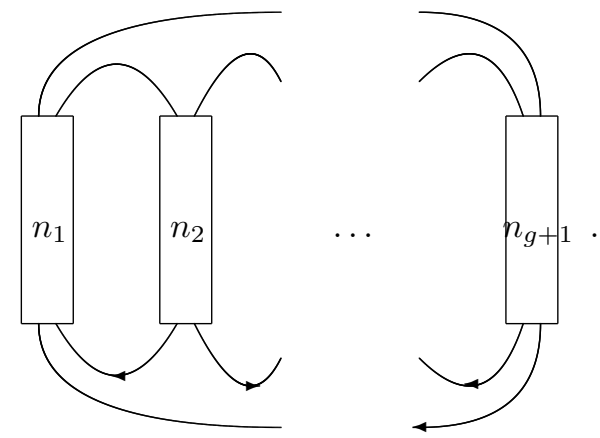
Имеем

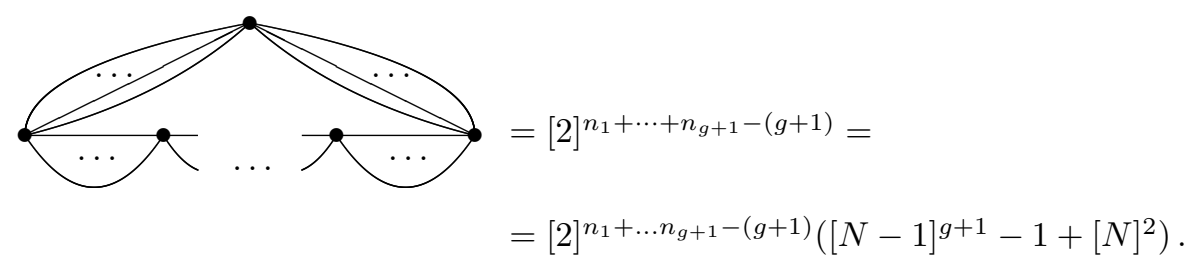

Для обычных (невиртуальных) узлов число вершин $g+1$ (где $g$ обозначает род поверхности, на которой лежит узел) четно, т. е. циклы имеют четные длины. Твистованные узлы $(2 g-2)_{1}$ дают частный пример этого случая, когда $n_{1}=\cdots=n_{g}=1$, a $n_{g+1}=2$.

Все антипараллельные узлы, две вершины, соединенные $g+1$ простым ребром:

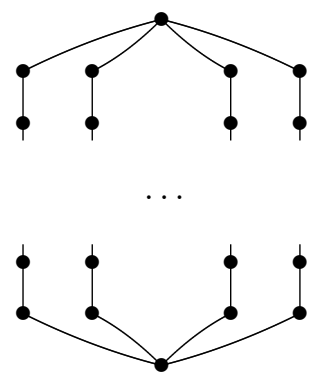

Для обычных (невиртуальных) узлов/зацеплений числа $n_{i}$ либо все нечетные, либо все четные; двухнитевые торические узлы/зацепления являются частным примером, когда $n_{i}=1$ для любого $i$.

Рассмотрим “четный” случай. Ответы для первых нескольких $g$ устроены так:

$$
\begin{aligned}
& f_{0}\left(x_{1}\right)=[N] x_{1}, \\
& f_{1}\left(x_{1}, x_{2}\right)=x_{1} x_{2}+y \\
& f_{2}\left(x_{1}, x_{2}, x_{3}\right)=\frac{1}{[N]}\left(x_{1} x_{2} x_{3}+y\left(x_{1}+x_{2}+x_{3}\right)+y^{2}\left(1-\frac{1}{y}\right)\right), \\
& f_{3}\left(x_{1}, x_{2}, x_{3}, x_{4}\right)=\frac{1}{[N]^{2}}\left(x_{1} x_{2} x_{3} x_{4}+y \sum_{i<j} x_{i} x_{j}+y^{2}\left(1-\frac{1}{y}\right) \sum_{i} x_{i}+\right. \\
& f_{4}\left(x_{1}, \ldots, x_{5}\right)=\frac{1}{[N]^{3}}\left(x_{1} x_{2} x_{3} x_{4} x_{5}+y \sum_{i<j<k} x_{i} x_{j} x_{k}+y^{2}\left(1-\frac{1}{y}\right) \sum_{i<j} x_{i} x_{j}+\right. \\
&\left.\quad+y^{3}\left(1-\frac{1}{y}+\frac{1}{y^{2}}\right) \sum_{i} x_{i}+y^{4}\left(1-\frac{1}{y}+\frac{1}{y^{2}}-\frac{1}{y^{3}}\right)\right)
\end{aligned}
$$

где $x_{i}=[N-1]^{n_{i}}$, а $y=[N-1][N+1]$. Мы надеемся, что общий ответ ясен из этих примеров (они написаны в терминах $q$-чисел с переменной $y$, играющей роль $q$ ). 
Рассмотрим "нечетные" циклы. Ответы для нескольких первых $g$ устроены следующим образом:

$$
\begin{aligned}
f_{0}\left(x_{1}\right) & =[N] x_{1}, \\
f_{1}\left(x_{1}, x_{2}\right) & =x_{1} x_{2}+y \\
f_{2}\left(x_{1}, x_{2}, x_{3}\right) & =\frac{1}{[N]}\left(x_{1} x_{2} x_{3}+y\left(x_{1}+x_{2}+x_{3}\right)+y^{2}\left(\frac{1}{[N-1]}-\frac{1}{[N+1]}\right)\right), \\
f_{3}\left(x_{1}, x_{2}, x_{3}, x_{4}\right) & =\frac{1}{[N]^{2}}\left(x_{1} x_{2} x_{3} x_{4}+y \sum_{i<j} x_{i} x_{j}+y^{2}\left(\frac{1}{[N-1]}-\frac{1}{[N+1]}\right) \sum_{i} x_{i}+\right. \\
& \left.+y^{3}\left(\frac{1}{[N-1]^{2}}-\frac{1}{[N-1][N+1]}+\frac{1}{[N+1]^{2}}\right)\right) .
\end{aligned}
$$

Общая формула должна быть ясна из этих примеров.

Претцелевы узлы рода $g=2$, две вершины, соединенные двумя мультиребрами и простой нечетной линией между ними:

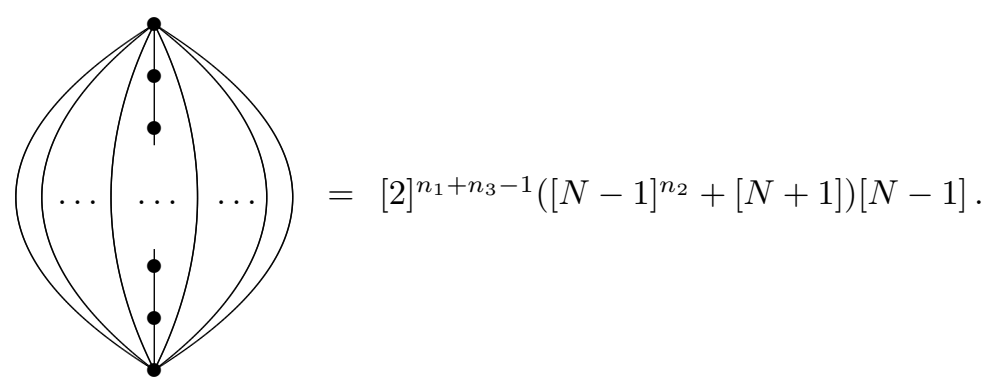

Множественности левого и правого мультиребер обозначены как $n_{1}$ и $n_{3}$ соответственно, а число ребер на центральной антипараллельной нечетной линии обозначено как $n_{2}$.

\section{B.3. Разное.}

В.3.1. Циклы. Циклы с нечетным и четным числом вершин равны соответственно

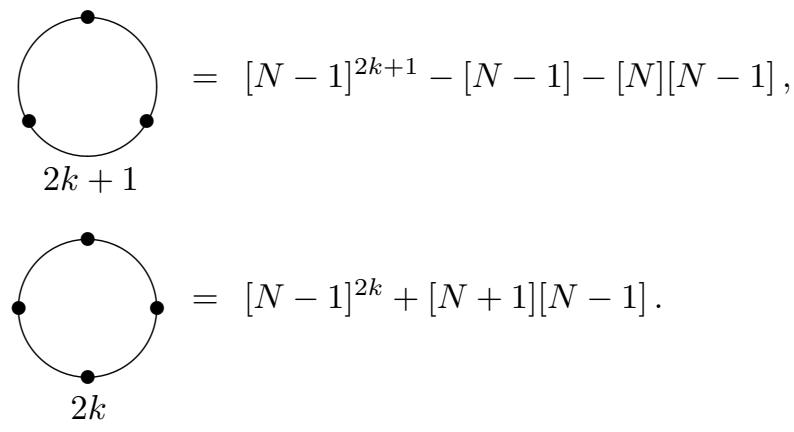


В.3.2. Простые линии. Простые линии из двухвалентных вершин с четным и нечетным числом внутренних вершин (обозначенных прямоугольником) равны соответственно

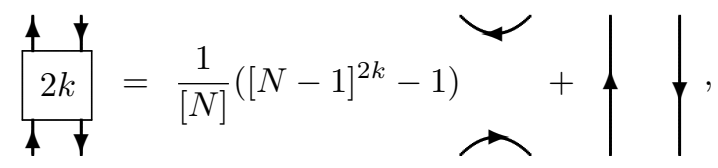

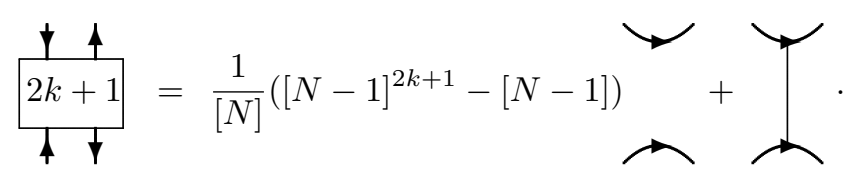

Благодарности. Мы благодарим Егора Зенкевича за стимулирующие обсуждения и особенно за идею рассмотреть высшие антисимметрические проекторы.

\section{Список литературы}

[1] V.F. R. Jones, "A polynomial invariant for knots via von Neumann algebras", Bull. Amer. Math. Soc. (N.S.), 12:1 (1985), 103-111; "On knot invariants related to some statistical mechanical models", Pacific J. Math., 137:2 (1989), 311-334; L. H. Kauffman, Knots and Physics, World Sci., Singapore, 1991; P. Freyd, D. Yetter, J. Hoste, W. B. R. Lickorish, K. Millett, A. Ocneanu, "A new polynomial invariant of knots and links", Bull. Amer. Math. Soc. (N.S.), 12:2 (1985), 239-246; J. H. Przytycki, K. P. Traczyk, "Invariants of links of Conway type", Kobe J. Math., 4:2 (1987), 115-139.

[2] S.-S. Chern, J. Simons, "Some cohomology classes in principal fiber bundles and their application to Riemannian geometry", Proc. Nat. Acad. Sci. U.S.A., 68:4 (1971), 791-794; A. S. Schwarz, "New topological invariants arising in the theory of quantized fields", Topology and Its Applications, Talk at the International Topological Conference. Abstracts. Part 2 (Baku, Azerbaijan, October 3-8, 1987), ed. S. P. Novikov, AMS, Providence, RI, 1993; E. Witten, "Quantum field theory and the Jones polynomial", Commun. Math. Phys., 121:3 (1989), 351-399; M. Atiyah, The Geometry and Physics of Knots, Cambridge Univ. Press, Cambridge, 1990.

[3] L. H. Kauffman, "Virtual knot theory", European J. Combin., 20:7 (1999), 663-690, arXiv: math/9811028; R. Fenn, D. P. Ilyutko, L. H. Kauffman, V. O. Manturov, "Unsolved problems in virtual knot theory and combinatorial knot theory", Knots in Poland III. Part III (Stefan Banach International Mathematical Center, Warsaw, Poland, July 18-25, 2010 and Bȩdlewo, Poland, July 25 - August 4, 2010), Banach Center Publications, 103, eds. J. H. Przytycki, P. Traczyk, Polish Acad. Sci. Inst. Math., Warszawa, 2014, 9-61, arXiv: 1409.2823 .

[4] M. Khovanov, "A categorification of the Jones polynomial", Duke Math. J., 101:3 (2000), 359-426, arXiv: math/9908171.

[5] M. Khovanov, L. Rozansky, "Matrix factorizations and link homology", Fund. Math., 199:1 (2008), 1-91, arXiv: math/0401268; "Matrix factorizations and link homology. II", Geom. Topol., 12:3 (2008), 1387-1425, arXiv: math/0505056; "Virtual crossings, convolutions and a categorification of the $\mathrm{SO}(2 N)$ Kauffman polynomial", J. Gökova Geom. Topol., 1 (2007), 116-214, arXiv: math/0701333; N. Carqueville, D. Murfet, "Computing Khovanov-Rozansky homology and defect fusion", Algebr. Geom. Topol., 14:1 (2014), 489-537, arXiv: 1108.1081. 
[6] S. Gukov, A. Schwarz, C. Vafa, "Khovanov-Rozansky homology and topological strings", Lett. Math. Phys., 74:1 (2005), 53-74, arXiv: hep-th/0412243; N. M. Dunfield, S. Gukov, J. Rasmussen, "The superpolynomial for knot homologies", Exp. Math., 15:2 (2006), 129-159, arXiv: math/0505662; M. Aganagic, Sh. Shakirov, Knot homology from refined Chern-Simons theory, arXiv: 1105.5117; "Refined Chern-Simons theory and knot homology", String-Math 2011, Proceedings of Symposia in Pure Mathematics, 85, eds. J. Block, J. Distler, R. Donagi, E. Sharpe, AMS, Providence, RI, 2012, 3-31, arXiv: 1202.2489; P. Dunin-Barkowski, A. Mironov, A. Morozov, A. Sleptsov, A. Smirnov, "Superpolynomials for torus knots from evolution induced by cut-and-join operators", JHEP, 03 (2013), 021, 85 pp., arXiv: 1106.4305; A. Mironov, A. Morozov, S. Shakirov, A. Sleptsov, "Interplay between MacDonald and Hall-Littlewood expansions of extended torus superpolynomials", JHEP, 05 (2012), 70, 11 pp., arXiv: 1201.3339; I. Cherednik, Jones polynomials of torus knots via DAHA, arXiv: 1111.6195; "DAHA-Jones polynomials of torus knots", Selecta Math. (N. S.), 22:2 (2016), 1013-1053, arXiv: 1406.3959; E. Gorsky, A. Oblomkov, J. Rasmussen, "On stable Khovanov homology of torus knots", Exp. Math., 22:3 (2013), 265-281, arXiv: 1206.2226; E. Gorsky, A. Negut, "Refined knot invariants and Hilbert schemes", J. Math. Pures Appl. (9), 104:3 (2015), 403-435, arXiv: 1304.3328; I. Cherednik, I. Danilenko, "DAHA and iterated torus knots", Algebr. Geom. Topol., 16:2 (2016), 843-898, arXiv: 1408.4348.

[7] M. Aganagic, A. Klemm, M. Mariño, C. Vafa, "The topological vertex", Commun. Math. Phys., 254:2 (2005), 425-478, arXiv: hep-th/0305132; A. Iqbal, C. Kozcaz, C. Vafa, "The refined topological vertex", JHEP, 10 (2009), 069, 58 pp.; S. Gukov, A. Iqbal, C. Kozçaz, C. Vafa, "Link homologies and the refined topological vertex", Commun. Math. Phys., 298:3 (2010), 757-785, arXiv:0705.1368; M. Taki, "Refined topological vertex and instanton counting", JHEP, 03 (2008), 048, 22 pp., arXiv: 0710.1776; H. Awata, H. Kanno, "Changing the preferred direction of the refined topological vertex", J. Geom. Phys., 64 (2013), 91-110, arXiv: 0903.5383; N. Nekrasov, A. Okounkov, "Membranes and sheaves", Algebr. Geom., 3:3 (2016), 320-369, arXiv: 1404.2323; Y. Zenkevich, "Generalized Macdonald polynomials, spectral duality for conformal blocks and AGT correspondence in five dimensions", JHEP, 05 (2015), 131, 22 pp., arXiv: 1412.8592.

[8] А. Ю. Морозов, “Загадки $\beta$-деформации”, ТМФ, 173:1 (2012), 104-126.

[9] E. Gorsky, S. Gukov, M. Stosic, Quadruply-graded colored homology of knots, arXiv: 1304.3481.

[10] S. Arthamonov, Sh. Shakirov, Refined Chern-Simons theory in genus two, arXiv: 1504.02620 .

[11] S. Kharchev, A. Marshakov, A. Mironov, A. Morozov, "Generalized Kazakov-Migdal-Kontsevich model: group theory aspects", Internat. J. Modern Phys. A, 10:14 (1995), 2015-2052; А. Д. Миронов, А. Ю. Морозов, С. М. Натанзон, "Полный набор операторов разрезания и склейки в теории Гурвица-Концевича", ТМФ, 166:1 (2011), 3-27, arXiv: 0904.4227; A. Mironov, A. Morozov, S. Natanzon, "Algebra of differential operators associated with Young diagrams", J. Geom. Phys., 62:2 (2012), 148-155, arXiv: 1012.0433; А. Д. Миронов, А. Ю. Морозов, А. В. Слепцов, "Разложение по родам для полиномов ХОМФЛИ", ТМФ, 177:2 (2013), 179-221, arXiv: 1303.1015; A. Mironov, A. Morozov, A. Sleptsov, "On genus expansion of knot polynomials and hidden structure of Hurwitz tau-functions", Eur. Phys. J. C, 73:7 (2013), 2492, arXiv: 1304.7499; A. Mironov, A. Morozov, A. Sleptsov, A. Smirnov, "On genus expansion of superpolynomials", Nucl. Phys. B, 889 (2014), 757-777, arXiv: 1310.7622; A. Alexandrov, A. Mironov, A. Morozov, S. Natanzon, "Integrability of Hurwitz partition functions", J. Phys. A: Math. Theor., 45:4 (2012), 045209, 10 pp., arXiv: 1103.4100; "On KP-integrable Hurwitz functions", JHEP, 11 (2014), 080, 30 pp., arXiv: 1405.1395.

[12] А. Ю. Морозов, “Теория струн - что это такое?", УФН, 162:8, 83-175; “Интегрируемость и матричные модели", УФН, 164:1 (1994), 3-62, arXiv: hep-th/9303139; A. Morozov, "Matrix models as integrable systems", Particles and Fields (Banff, Canada, 16-24 
August, 1994), CRM Series in Mathematical Physics, eds. G. W. Semenoff, L. Vinet, Springer, New York, 1999, 127-210, arXiv: hep-th/9502091; "Challenges of matrix models", String Theory: From Gauge Interactions to Cosmology, NATO Science Series II: Mathematics, Physics and Chemistry, 208, eds. L. Baulieu, J. de Boer, B. Pioline, E. Rabinovici, Springer, Dordrecht, 2006, 129-162, arXiv: hep-th/0502010; " $2 d$ gravity and matrix models. I. $2 d$ gravity", Internat. J. Modern Phys. A, 9:25 (1994), 4355-4405, arXiv: hep-th/9312212; А. Д. Миронов, "Матричные модели двумерной гравитации", ЭЧАЯ, $33: 5$ (2002), 1051-1145; "Матричные модели и матричные интегралы", ТМФ, 146:1 (2006), 77-89, arXiv: hep-th/0506158.

[13] A. Alexandrov, A. Mironov, A. Morozov, "Partition functions of matrix models: first special functions of string theory", Internat. J. Modern Phys. A, 19:24 (2004), 4127-4165, arXiv: hep-th/0310113; "Unified description of correlators in non-gaussian phases of hermitian matrix model", Internat. J. Modern Phys. A, 21:12 (2006), 2481-2517, arXiv: hep-th/0412099; "Unified description of correlators in non-Gaussian phases of hermitian matrix model", Internat. J. Modern Phys. A, 21:12 (2006), 2481-2518, arXiv: hep-th/0412099; "Solving Virasoro constraints in matrix models", Fortsch. Phys., 53:5-6 (2005), 512-521, arXiv: hep-th/0412205; "Instantons and merons in matrix models", Phys. D, 235:1-2 (2007), 126-167, arXiv: hep-th/0608228; "BGWM as second constituent of complex matrix model", JHEP, 12 (2009), 053, 49 pp., arXiv: 0906.3305; А. С. Александров, А. Д. Миронов, А. Ю. Морозов, “M-теория матричных моделей”, ТМФ, 150:2 (2007), 179-192, arXiv: hep-th/0605171; L. Chekhov, B. Eynard, N. Orantin, "Free energy topological expansion for the 2-matrix model", JHEP, 12 (2006), 053, 31 pp., arXiv: math-ph/0603003; B. Eynard, N. Orantin, "Invariants of algebraic curves and topological expansion", Commun. Number Theory Phys., 1:2 (2007), 347-452, arXiv: math-ph/0702045; A. Alexandrov, A. Mironov, A. Morozov, P. Putrov, "Partition functions of matrix models as the first special functions of string theory II: Kontsevich model", Internat. J. Modern Phys. A, 24:27 (2009), 4939-4998, arXiv: 0811.2825.

[14] J. C. Baez, J. Dolan, "Categorification", Higher Category Theory (Northwestern University, Evanston, IL, March 28-30, 1997), Contemporary Mathematics, 230, eds. E. Getzler, M. Kapranov, AMS, Providence, RI, 1998, 1-36, arXiv: math.QA/9802029; L. Crane, D. Yetter, "Examples of categorification", Cah. Topol. Géom. Différ. Catég., 39:1 (1998), 3-25; V. Mazorchuk, Lectures on Algebraic Categorification, QGM Master Class Series, EMS, Zürich, 2012; A. Savage, Introduction to categorification, arXiv: 1401.6037; M. Khovanov, V. Mazorchuk, C. Stroppel, "A brief review of abelian categorifications", Theory Appl. Categ., 22:19 (200), 479-508, arXiv: math.RT/0702746.

[15] E. Witten, "Supersymmetry and Morse theory", J. Differential Geom., 17:4 (1982), 661-692; "Two lectures on the Jones polynomial and Khovanov homology", Proceedings of the Freedman Fest (Santa Barbara, CA, USA, April 15-17, 2011; Berkeley, CA, USA, June 6-10, 2011), Geometry and Topology Monographs, 18, eds. R. Kirby, V. Krushkal, Z. Wang, Geom. Topol. Publ., Coventry, 2012, 291-308, arXiv: 1401.6996; A. Kapustin, E. Witten, "Electric-magnetic duality and the geometric Langlands Program", Commun. Number Theory Phys., 1:1 (2007), 1-236, arXiv: hep-th/0604151.

[16] L. H. Kauffman, "State models and the Jones polynomial", Topology, 26:3 (1987), 395-407; "Invariants of graphs in three-space", Trans. Amer. Math. Soc., 311:2 (1989), 697-710; L. H. Kauffman, P. Vogel, "Link polynomials and a graphical calculus", J. Knot Theory Ramifications, 1:1 (1992), 59-104.

[17] M. Khovanov, "Patterns in knot cohomology. I", Exp. Math., 12:3 (2003), 365-374, arXiv: math/0201306; "Categorifications of the colored Jones polynomial", J. Knot Theory Ramifications, 14:1 (2005), 111-130, arXiv: math/0302060; "sl(3) link homology", Algebr. Geom. Topol., 4 (2004), 1045-1081, arXiv: math/0304375; "Triply-graded link homology and Hochschild homology of Soergel bimodules", Internat. J. Math., 18:8 (2007), 869-885, arXiv: math/0510265; "Link homology and categorification", Proceedings of the International Congress of Mathematicians, v. 2 (Madrid, 22-30 August, 2006), eds. M. Sanz-Solé, 
J. Soria, J. L. Varona, J. Verdera, Eur. Math. Soc., Zürich, 989-999, arXiv: math/0605339; M. Khovanov, "Categorifications from planar diagrammatics", Japanese J. Math., 5:2 (2010), 153-181, arXiv: 1008.5084.

[18] D. Bar-Natan, "On Khovanov's categorification of the Jones polynomial", Algebr. Geom. Topol., 2:1 (2002), 337-370, arXiv: math/0201043; "Khovanov's homology for tangles and cobordisms", Geom. Topol., 9:3 (2005), 1443-1499, arXiv: math/0410495; D. Bar-Natan, "Fast Khovanov homology computations", J. Knot Theory Ramifications, 16:3 (2007), 243-255, arXiv: math/0606318.

[19] V. Dolotin, A. Morozov, "Introduction to Khovanov homologies. I. Unreduced Jones superpolynomial", JHEP, 01 (2013), 065, 46 pp., arXiv: 1208.4994; "Introduction to Khovanov homologies. II. Reduced Jones superpolynomials", J. Phys.: Conf. Ser., 411:1 (2013), 012013, 21 pp., arXiv: 1209.5109.

[20] V. Dolotin, A. Morozov, "Introduction to Khovanov homologies. III. A new and simple tensor-algebra construction of Khovanov-Rozansky invariants", Nucl. Phys. B, 878 (2014), 12-81, arXiv: 1308.5759 .

[21] A. Morozov, And. Morozov, A. Popolitov, "On matrix-model approach to simplified Khovanov-Rozansky calculus", Phys. Lett. B, 749 (2015), 309-325, arXiv: 1506.07516.

[22] V. Dolotin, A. Morozov, Introduction to Non-Linear Algebra, World Sci., Singapore, 2007, arXiv: hep-th/0609022.

[23] A. Anokhina, A. Morozov, "Towards $\mathcal{R}$-matrix construction of Khovanov-Rozansky polynomials. I. Primary T-deformation of HOMFLY", JHEP, 07 (2014), 063, 180 pp., arXiv: 1403.8087 .

[24] A. Morozov, And. Morozov, Ant. Morozov, "On possible existence of HOMFLY polynomials for virtual knots", Phys. Lett. B, 737 (2014), 48-56, arXiv: 1407.6319; L. Bishler, A. Morozov, An. Morozov, Ant. Morozov, "Evolution method and HOMFLY polynomials for virtual knots", Internat. J. Modern Phys. A, 30:14 (2015), 1550074, 39 pp., arXiv: 1411.2569.

[25] N. Yu. Reshetikhin, V. G. Turaev, "Ribbon graphs and their invaraints derived from quantum groups", Commun. Math. Phys., 127:1 (1990), 1-26; E. Guadagnini, M. Martellini, M. Mintchev, "Chern-Simons field theory and quantum groups", Quantum Groups, Proceedings of the 8th International Workshop on Mathematical Physics (Clausthal, Germany, July 19-26, 1989), Lecture Notes in Physics, 370, eds. H. D. Doebner, J. D. Hennig, World Sci., Singapore, 1990, 307-317; "Chern-Simons holonomies and the appearance of quantum groups", Phys. Lett. B, 235:3-4 (1990), 275-281.

[26] R. K. Kaul, T. R. Govindarajan, "Three-dimensional Chern-Simons theory as a theory of knots and links", Nucl. Phys. B, 380:1-2 (1992), 293-333, arXiv: hep-th/9111063; "Three-dimensional Chern-Simons theory as a theory of knots and links: (II). Multicoloured links", 393:1-2 (1993), 392-412; P. Rama Devi, T. R. Govindarajan, R. K. Kaul, "Three-dimensional Chern-Simons theory as a theory of knots and links. (III). Compact semi-simple group", Nucl. Phys. B, 402:1-2 (1993), 548-566, arXiv: hep-th/9212110; "Knot invariants from rational conformal field theories", Nucl. Phys. B, 422:1-2 (1994), 291-306, arXiv: hep-th/9312215; "Representations of composite braids and invariants for mutant knots and links in Chern-Simons field theories", Modern Phys. Lett. A, 10:22 (1995), 1635-1658, arXiv: hep-th/9412084; Zodinmawia, P. Ramadevi, "SU $N)$ quantum Racah coefficients and non-torus links", Nucl. Phys. B, 870:1 (2013), 205-242, arXiv: 1107.3918; Reformulated invariants for non-torus knots and links, arXiv: 1209.1346; D. Galakhov, D. Melnikov, A. Mironov, A. Morozov, A. Sleptsov, "Colored knot polynomials for arbitrary Pretzel knots and links", Phys. Lett. B, 743 (2015), 71-74, arXiv: 1412.2616; A. Mironov, A. Morozov, A. Sleptsov, "Colored HOMFLY polynomials for the pretzel knots and links", JHEP, 07 (2015), 069, 34 pp., arXiv: 1412.8432; D. Galakhov, D. Melnikov, A. Mironov, A. Morozov, A. Sleptsov, "Knot invariants from Virasoro related representation and Pretzel knots", Nucl. Phys. B, 899 (2015), 194-228, arXiv: 1502.02621; S. Nawata, P. Ramadevi, V.K. Singh, Colored HOMFLY polynomials that distinguish mutant knots, arXiv: 
1504.00364; A. Mironov, A. Morozov, And. Morozov, P. Ramadevi, V. K. Singh, JHEP, 07 (2015), 109, 68 pp., arXiv: 1504.00371.

[27] A. Morozov, A. Smirnov, "Chern-Simons teory in the temporal gauge and knot invariants through the universal quantum $R$-matrix", Nucl. Phys. B, 835:3 (2010), 284-313, arXiv: 1001.2003; A. Smirnov, "Notes on Chern-Simons theory in the temporal gauge", Proceedings of 47th International School of Subnuclear Physics (Erice, Italy, 29 August -7 September, 2009 Sicily), ed. A. Zichichi, World Sci., Singapore, 2012, 489-498, arXiv: 0910.5011; A. Mironov, A. Morozov, And. Morozov, "Character expansion for HOMFLY polynomials. II. Fundamental representation. Up to five strands in braid", JHEP, 03 (2012), 034, 33 pp., arXiv: 1112.2654; "Character expansion for HOMFLY polynomials I. Integrability and difference equations", Strings, Gauge Fields, and the Geometry Behind (The Legacy of Maximilian Kreuzer), eds. A. Rebhan, L. Katzarkov, J. Knapp, R. Rashkov, E. Scheidegger, World Sci., Singapore, 2013, 101-118, arXiv: 1112.5754; A. Mironov, A. Morozov, And. Morozov, "Evolution method and 'differential hierarchy' of colored knot polynomials", AIP Conf. Proc., 1562:1 (2013), 123-155, arXiv: 1306.3197; A. Anokhina, A. Mironov, A. Morozov, And. Morozov, "Colored HOMFLY polynomials as multiple sums over paths or standard Young tableaux", Adv. High Energy Phys., 2013, 931830, 12 pp., arXiv: 1304.1486; С. Б. Артамонов, А. Д. Миронов, А. Ю. Морозов, "Иерархия дифференциалов и дополнительная градуировка полиномов узлов", ТMФ, 179:2 (2014), 147-188, arXiv: 1306.5682 ; А. С. Анохина, А. А. Морозов, "Процедура каблирования для раскрашенных полиномов ХОМФЛИ", ТМФ, 178:1 (2014), 3-68, arXiv: 1307.2216; Ya. Kononov, A. Morozov, "On the defect and stability of differential expansion", Писъма в ККЭТФ, 101:12 (2015), 931-934, arXiv: 1504.07146; "Factorization of colored knot polynomials at roots of unity", Phys. Lett. B, 747 (2015), 500-510, arXiv: 1505.06170; A. Mironov, A. Morozov, "Towards effective topological field theory for knots", Nucl. Phys. B, 899 (2015), 395-413, arXiv: 1506.00339.

[28] O.T. Dasbach, A.M. Lowrance, "A Turaev surface approach to Khovanov homology", Quantum Topol., 5:4 (2014), 425-486, arXiv: 1107.2344.

[29] A. Brini, B. Eynard, M. Mariño, "Torus knots and mirror symmetry", Ann. Henri Poincaré, 13:8 (2012), 1873-1910, arXiv: 1105.2012; A. Aleksandrov, A. D. Mironov, A. Morozov, A. A. Morozov, "Towards matrix model representation of HOMFLY polynomials", Писъма в ЖКЭТФ, 100:4 (2014), 297-304; J. Gu, A. Klemm, M. Mariño, J. Reuter, "Exact solutions to quantum spectral curves by topological string theory", JHEP, 10 (2015), 025, 68 pp., arXiv: 1506.09176.

[30] P. Cvitanović, Group Theory: Birdtracks, Lie's, and Exceptional Groups, Princeton Univ. Press, Princeton, NJ, 2008. 